University of Nebraska - Lincoln

DigitalCommons@University of Nebraska - Lincoln

Supply Chain Management and Analytics

Publications

Business, College of

2019

Dynamic Pricing of Wireless Internet Based on Usage and Stochastically Changing Capacity

Demet Batur

Jennifer K. Ryan

Zhongyuan Zhao

Mehmet C. Vuran

Follow this and additional works at: https://digitalcommons.unl.edu/supplychain

Part of the Business Administration, Management, and Operations Commons, Management Information Systems Commons, Management Sciences and Quantitative Methods Commons, Operations and Supply Chain Management Commons, and the Technology and Innovation Commons

This Article is brought to you for free and open access by the Business, College of at DigitalCommons@University of Nebraska - Lincoln. It has been accepted for inclusion in Supply Chain Management and Analytics Publications by an authorized administrator of DigitalCommons@University of Nebraska - Lincoln. 


\section{Published as:}

Batur, D., J.K. Ryan, M.C. Vuran and Z. Zhao, "Dynamic Pricing of Wireless Internet Based on Usage and Stochastically Changing Capacity". Manufacturing \& Service Operations Management. DOI:10.1287/msom.2018.0727 (2019).

Volume 21, Issue 4 (Fall 2019)

https://pubsonline.informs.org/doi/10.1287/msom.2018.0727

ORCID

Demet Batur https://orcid.org/0000-0001-5486-6799

Jennifer K. Ryan https://orcid.org/0000-0002-8045-8614

Zhongyuan Zhao https://orcid.org/0000-0003-0346-8015

Mehmet C. Vuran https://orcid.org/0000-0001-7894-6611

Copyright (C) 2019, INFORMS.

Used by permission. 


\title{
Dynamic Pricing of Wireless Internet Based on Usage and Stochastically Changing Capacity
}

\author{
Demet Batur ${ }^{\mathrm{a}}$, Jennifer K. Ryan ${ }^{\mathrm{a}}$, Zhongyuan Zhao ${ }^{\mathrm{b}}$, Mehmet C. Vuran ${ }^{\mathrm{b}}$ \\ ${ }^{a}$ Department of Supply Chain Management and Analytics, University of Nebraska-Lincoln, Lincoln, NE 68588 \\ ${ }^{\mathrm{b}}$ Department of Computer Science and Engineering, University of Nebraska-Lincoln, Lincoln, NE 68588 \\ dbatur@unl.edu (DB), jennifer.ryan@unl.edu (JKR), zhzhao@cse.unl.edu (ZZ), mcvuran@cse.unl.edu (MCV)
}

(1) Problem Definition: Inspired by new developments in dynamic spectrum access, we study the dynamic pricing of wireless Internet access when demand and capacity (bandwidth) are stochastic. (2) Academic / Practical Relevance: The demand for wireless Internet access has increased enormously. However, the spectrum available to wireless service providers is limited. The industry has thus altered conventional licensebased spectrum access policies through unlicensed spectrum operations. The additional spectrum obtained through these operations has stochastic capacity. Thus, the pricing of this service by the service provider has novel challenges. The problem considered in this paper is therefore of high practical relevance and new to the academic literature. (3) Methodology: We study this pricing problem using a Markov Decision Process model in which customers are posted dynamic prices based on their bandwidth requirement and the available capacity. (4) Results: We characterize the structure of the optimal pricing policy as a function of the system state and of the input parameters. Since it is impossible to solve this problem for practically large state spaces, we propose a heuristic dynamic pricing policy that performs very well, particularly when the ratio of capacity to demand rate is low. (5) Managerial Implications: We demonstrate the value of using a dynamic heuristic pricing policy compared to the myopic and optimal static policies. The previous literature has studied similar systems with fixed capacity and has characterized conditions under which myopic policies perform well. In contrast, our setting has dynamic (stochastic) capacity, and we find that identifying good state-dependent heuristic pricing policies is of greater importance. Our heuristic policy is computationally more tractable, and easier to implement, than the optimal dynamic and static pricing policies. It also provides a significant performance improvement relative to the myopic and optimal static policies when capacity is scarce, a condition that holds for the practical setting which motivated this research.

Key words: dynamic pricing, wireless Internet, Markov Decision Process, stochastic capacity

History: Received: Jan 09, 2017; Accepted: Mar 10, 2018

\section{Introduction}

Wireless networks have revolutionized public life, especially following the invention of smartphones in 2007. It is estimated that by 2020, mobile data traffic will grow by a factor of ten, with a 1000-fold increase in demand in certain urban areas (Beltran et al. 2016), while the number of connected mobile devices is expected to reach 116 billion (Cisco 
VNI 2016, Feb. 3). These increased demands place a significant burden on mobile service providers (SPs) because of the scarcity of available wireless spectrum.

Today's wireless networks are characterized by a fixed spectrum assignment policy, where the resource, i.e., the spectrum, is allocated to users, such as government and broadcasters, in a license-based manner according to national policies, technical characteristics, and international agreements. While SPs try to utilize their share, a large portion of the assigned spectrum is used sporadically, and geographical variations in the utilization of assigned spectrum range from $15 \%$ to $85 \%$ (FCC Report 2002). This leads to the spectrum crisis, in which access policies to spectrum are questioned, e.g., Chen (2012, April 17).

The wireless industry has been altering conventional license-based spectrum access policies through unlicensed spectrum operational approaches. The most prominent solution has been WiFi, which exclusively operates on unlicensed spectrum (e.g., $2.4 \mathrm{GHz}, 5 \mathrm{GHz}$ ). More recently, the spectrum crisis has forced government agencies to adopt unlicensed spectrum operational approaches for other purposes. This leads to dynamic spectrum access (DSA), in which resources change dynamically with respect to the usage of the shared, unlicensed spectrum by several users. Examples of unlicensed spectrum operation are:

$T V$ White Space: TV channels have traditionally been allocated to broadcast companies throughout the nation. Due to population density dynamics, not all TV channels are used in all locations, e.g., while broadcast companies use all TV channels in locations such as Manhattan, few channels are used in less populated cities or rural areas. This leaves unused TV channels in certain areas, referred to as $T V$ white spaces, which can be repurposed for other wireless communication. In 2008, the Federal Communications Commission (FCC) allowed unlicensed devices, i.e., Television Band Devices (TVBDs), to operate in TV white spaces in the US (FCC-10-174 2010). TVBDs operate on available TV bands based on information received from TV spectrum databases or local spectrum sensing operations. Sites such as Google Spectrum and Microsoft Whitespaces have established nation-wide spectrum databases which dynamically allocate spectrum for unlicensed usage.

$T V$ Black Space: While suitable for rural areas, the FCC's current approach leaves populated urban areas, where the spectrum crisis is most significant, with few TV white spaces (Harrison et al. 2010). For example, 4 out of the 5 largest cities by population have less than $4 \mathrm{TV}$ white space channels. Thus, recent research studies have focused on finer-grade DSA schemes. Accordingly, an unlicensed user can access a TV channel if none 
Batur et al.: Dynamic Pricing of Wireless Internet

Article submitted to Manufacturing $\&$ Service Operations Management; manuscript no. (Please, provide the manuscript number!) 3

of the TV receivers in its immediate vicinity is watching that channel. Hence, the spectrum can be accessed based on dynamic behaviors of licensed users, i.e., TV viewers (Zhao et al. 2014, Zhang and Knightly 2015). Zhao et al. (2014) has shown that spectrum access based on TV viewership leads to up to a 5-fold increase in available capacity in New York.

3.5 GHz Citizens Broadband Radio Service (CBRS): In 2015, the FCC adopted a spectrum sharing policy to make $150 \mathrm{MHz}$ of spectrum available for mobile usage in the $3.5 \mathrm{GHz}$ band previously allocated to the U.S. Department of Defense (FCC-15-47 2015), using a three-tiered sharing mechanism: At the top tier, incumbent users, e.g., federal operations and satellite services, have exclusive rights to the spectrum in their vicinity. The priorityaccess tier allows use of part of the spectrum in a census track for a limited amount of time. The general authorized-access tier may use the remaining spectrum at no cost.

LTE Unlicensed: Initially spearheaded by the LTE-U Forum, SPs have begun standardizing $4 \mathrm{G}$ network operation on the unlicensed $5 \mathrm{GHz}$ band, to provide cellular networks access to additional unlicensed spectrum by sharing the spectrum with WiFi networks and other LTE operators. LTE-U would allow $4 \mathrm{G}$ networks to utilize the $5 \mathrm{GHz} \mathrm{WiFi}$ band when network demand exceeds available licensed resources (LTE-U 2015). LTE-U tries to utilize unused $5 \mathrm{GHz}$ channels, based on WiFi and LTE measurements. This results in three types of channels: (1) if a channel is unused by WiFi, the full capacity of that channel may be available to the SP for a certain duration; (2) if a channel is unused by WiFi, the capacity may be shared by two or more SPs; (3) if a channel is used by WiFi, LTE-U will share the channel with the WiFi network (Bhattarai et al. 2016).

These emerging DSA solutions result in the following challenges to network operators:

- Non-exclusive use: DSA approaches are characterized by the operator's use of unlicensed channels, which, by definition, are not exclusively reserved. As a result, unlicensed use of the spectrum provides capacity to the operator in a non-deterministic fashion.

- Dynamic Channel Availability: The availability of an unlicensed channel depends on factors that cannot be controlled by the network operator. For example, in TV white space, TV black space, and CBRS, the number of available channels changes with time depending on the behaviors of licensed users (i.e., TV viewers or federal operations).

- Varying Channel Capacity: In DSA solutions, two available channels may not provide the same capacity. For example, in LTE-U, if an unused $5 \mathrm{GHz}$ channel is found, the operator can utilize the maximum capacity provided by that channel. However, in some cases, the 


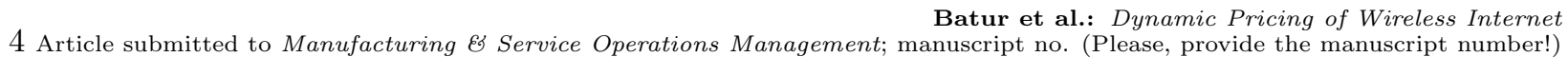

channel capacity may need to be shared with a WiFi network, leading to a lower, and dynamically changing, available capacity. As a result, different types of channel classes exist due to their available capacity, as well as the amount of time they are available to the network operator.

These three factors imply that the capacity of the wireless network will vary dynamically. In addition, it is highly likely that the capacity will not be sufficient to provide a good quality Internet service to users at the peak hours of demand, especially in urban areas. To be able to manage the peak-hour demand, we propose to use a dynamic pricing scheme in which the fees charged for an Internet connection will change in real-time.

To study this problem, we consider a network in which an SP monitors the availability of unlicensed channels and posts prices to the incoming customers based on the available bandwidth. If an arriving customer is willing to accept the posted price, he will start his Internet connection; otherwise, he will depart from the system. We consider a system with multiple classes of customers, where classes have different bandwidth requirements based on the type of activity to be performed, e.g., video streaming vs. web surfing vs. checking email. The connection fee paid by an arriving customer depends on the posted price at the time of arrival and the connection duration. A key feature of this setting is that the available bandwidth is a stochastic process due to the fact that unlicensed channels become available for Internet use if they are not actively used by licensed users, and they become unavailable when licensed users start using the channel. This implies that the available bandwidth may decrease at random, and thus customers that are in service may need to be dropped by the SP. A key challenge in managing the network is to maximize the revenue earned from admitted customers, while limiting the cost of dropped customers.

For this setting, we develop an optimal dynamic pricing strategy to maximize the revenue earned by an SP using DSA policies on unlicensed bands, while considering the limited capacity, as well as the potential losses associated with dropped customers. We model the system as a Markov decision process (MDP) in which customers arrive as a stochastic process, with stochastic service times, and unlicensed channels become available (providing bandwidth) as a stochastic process, with stochastic times until the channel becomes unavailable. Thus, the state of the system includes both the number of customers of each class being served and the number of channels of each class available for use by the system. Due to the high-dimension state, the optimal policy is difficult to compute and impractical 
Batur et al.: Dynamic Pricing of Wireless Internet

Article submitted to Manufacturing $\&$ Service Operations Management; manuscript no. (Please, provide the manuscript number!) 5

to implement. Therefore, we develop several heuristic policies in which the customer classes and/or channel classes are aggregated. We use a comprehensive set of numerical experiments to demonstrate that the proposed heuristic performs well with respect to the optimal policy. We also compare the performance of the proposed heuristic, which is dynamic (i.e., state-dependent), with non-state-dependent (i.e., static) pricing policies. We find that the proposed heuristic performs best, particularly when the ratio of capacity to demand rate is low, a condition which holds for the practical setting which motivated this research.

\section{Literature Review}

The research in this paper has some similarity to the previous literature in two areas: (1) dynamic pricing in MDP models and (2) admission control in systems with multiple classes of demand and stochastic service rates. A key distinguishing feature of our model, compared to this previous work, is that in addition to having stochastic inter-arrival and service times, our model also has stochastic capacity availability.

The most closely related previous work is that of Gans and Savin (2007), henceforth GS, who focus on dynamic pricing of rental assets (e.g., cars) when there is stochastic demand from two classes of customers, i.e., contract customers (who may be admitted at a pre-negotiated price, or rejected) and walk-in customers (who are quoted dynamic prices based on the number of customers already in the system). GS focus on characterizing the conditions under which myopic pricing policies will be optimal for certain customer classes. While some of our analysis builds on GS, there are a number of key differences:

- In GS, all customers have the same capacity requirement, which is normalized to one unit (e.g., every customer wants to rent a single car), while the total available capacity (e.g., rental car fleet size) is fixed and known. In contrast, in our model, the capacity requirement varies by customer class, but is exogenously specified, while the total available capacity evolves as a stochastic process as channels become available or unavailable.

- In our model, capacity may decrease, potentially requiring the SP to drop customers from service, at a cost. Thus, we must determine a drop-off policy, which specifies the set of customers to be dropped in a given state, in addition to a dynamic pricing policy.

- GS make admission decisions for contract customers, and pricing decisions for walk-in customers, where the latter is based on the class of the arriving customer and the current number of customers of each class in the system. In our model, there are no contract 


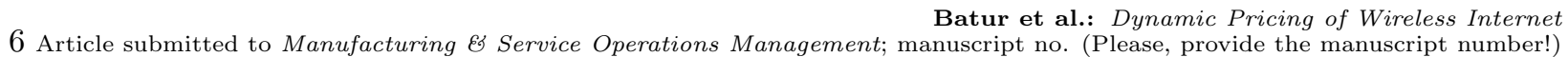
customers, and our dynamic pricing decision is based on the class of the arriving customer, the number of customers of each class in the system, and the current available capacity.

- GS focus on characterizing conditions under which preferred customer classes will exist and for which myopic policies will perform optimally or close-to-optimally. In contrast, we focus on developing heuristic policies that are not myopic, i.e., which are state-dependent.

Other research considers dynamic pricing and/or admission control for network services in which an SP has available capacity which may be used to satisfy the demands of customers who arrive stochastically and have class-dependent capacity requirements. This previous work takes the SP's total available capacity as fixed and known. For example, Paschalidis and Tsitsiklis (2000) consider dynamic pricing policies in such settings. They study the case in which the available capacity is large and there are many users with very small capacity requirements. They demonstrate that a static pricing policy is optimal in the limit. Mutlu et al. (2009) consider a setting with fixed capacity and two customer classes (primary and secondary users), where each class has the same service rate and each customer uses one unit of capacity. The SP makes pricing decisions for arriving secondary users, where the price depends on the number of customers in the system. Other literature on network services with stochastic arrivals considers admission control with exogenouslyspecified prices (Zhao et al. (2010), Turhan et al. (2012), Yahav et al. (2013)).

Another class of relevant research considers queuing models of admission control problems in loss systems with multiple classes of demand. In much of this work, the resource or capacity requirement (e.g., number of servers) is the same (and generally normalized to one) for all customer classes, e.g., Ulukus et al. (2011), Turhan et al. (2012), and Carrizosa et al. (1998). Since our model may require the dropping of customers who have been admitted to the system, the most relevant papers are those that consider preemption or termination of a customer, including Turhan et al. (2012) and Ulukus et al. (2011). However, in contrast to these papers, our model allows the bandwidth requirement per customer to vary by customer class. Thus, it is most similar to the literature on stochastic knapsacks, in which there are a fixed number of servers, with the number of servers required per customer varying by customer class, e.g., Altman et al. (2001) and Ross and Tsang (1989). Our model also has similarities to the literature on loss systems with batch arrivals, including Örmeci and Burnetas (2004). However, all of the above referenced works assume that the total available capacity (number of servers) is fixed and known. Further, while 
Batur et al.: Dynamic Pricing of Wireless Internet

Article submitted to Manufacturing $\&$ Service Operations Management; manuscript no. (Please, provide the manuscript number!) 7

these papers consider the admission control problem, we manage admission and maximize expected discounted profit by setting the optimal price for each arriving customer.

There is also recent literature that considers service systems that use self-scheduling servers, e.g., drivers in ride-sharing companies or agents in work-from-home call centers. These self-scheduling servers are not employees in the traditional sense and their schedules are not dictated by the company, i.e., the servers determine their own work schedules. In such business environments, as in the problem setting that we consider, both customer demand and service capacity change stochastically. Hence, to better match supply and demand, dynamic pricing may be used to manage demand (i.e., the customers). Alternatively, dynamic wages can be used to manage supply (i.e., the servers). In particular, surge pricing is often used as a tool to motivate servers to show up to work at required times. Cachon et al. (2017), Gurvich et al. (2017), and Ibrahim (2017) study the role of dynamic wages in such systems. Our paper is different from this line of work in three ways: i) These works try to match capacity, i.e., the number of self-scheduling servers, to demand while we try to match demand to capacity using admission control through pricing. ii) In these works, each customer is served by a single server (i.e., single unit of capacity), while in our model the customers' bandwidth (i.e., capacity) requirements vary by customer class. iii) In these works, a server cannot terminate a customer's service before service is completed. Thus, in contrast to our setting, customer drop-off is not a concern. There is also recent work on time-dependent pricing schemes in wireless networks (Zhang et al. 2014, Chang \begin{tabular}{|l|l|l|l|}
\hline et al. 2015, Huang et al. & 2016, Tsai et al. 2017). In these schemes, data pricing for the
\end{tabular} next day is determined based on information from the previous day(s). Prices are allowed to vary from one time-slot to another (e.g., hourly) to encourage users to shift demand from peak to off-peak hours. However, prices are not adjusted in real-time based on the system state, and the network capacity is assumed to be fixed and known.

\section{Problem Formulation}

We next introduce our model for the SP's problem. The notation and model formulation used in this paper are similar to GS. However, our model and analysis are more complex due to the fact that capacity is a stochastic process, rather than constant.

\subsection{Model Assumptions and Notation}

In this section, we first outline our assumptions and notation for the customer and channel classes. We then describe the overall system state and how it evolves over time. 


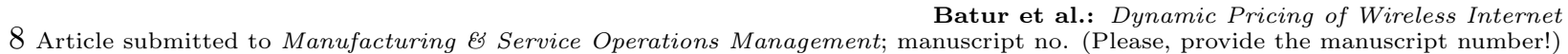

3.1.1. Customer Classes There are $M$ customer classes, indexed by $i=1,2, \ldots, M$. Customers in class $i$ arrive according to a Poisson process with rate $\lambda_{i}$. The service time for class $i$ customers is exponentially distributed with rate $\mu_{i}$. The bandwidth (capacity) requirement for a class $i$ customer is $r_{i}$, where $0<r_{i}<1$. For convenience, we define $\boldsymbol{\mu}=\left(\mu_{1}, \ldots, \mu_{M}\right), \mathbf{r}=\left(r_{1}, \ldots, r_{M}\right)$, and $\boldsymbol{\lambda}=\left(\lambda_{1}, \ldots, \lambda_{M}\right)$. In general, we use bold to denote vectors. The customer classes are ordered according to the following assumption.

Assumption 1. $r_{M} \leq r_{M-1} \leq \cdots \leq r_{2} \leq r_{1}<1$

We standardize the total bandwidth provided by a single channel (of any class) to 1. Thus, since $r_{i}<1$, several customers can be simultaneously served by a single channel.

The number of class $i$ customers in the system at time $t$ is denoted by $N_{i}(t)$. We let $\mathbf{N}(t)=\left(N_{1}(t), \ldots, N_{M}(t)\right)$ and we define $N(t)=\sum_{i=1}^{M} N_{i}(t)$. We will drop the argument $t$ when it is not required for clarity.

The SP must select the price to post to each arriving customer. The price set for Internet usage per unit of time is $\mathcal{U}=\left\{u_{1}, \ldots, u_{L}\right\}$, where the prices are ordered such that $u_{1}<u_{2}<$ $\ldots<u_{L}$. Since each customer class has a different bandwidth requirement, $r_{i}$, we scale the price set $\mathcal{U}$ by $r_{i}$ to find the price set for customer class $i$. For example, the set of prices that can be posted to an arriving class $i$ customer, denoted by $\mathcal{U}_{i}=\left\{u_{i 1}, \ldots, u_{i L}\right\}$, may be obtained by setting $u_{i \ell}=r_{i} u_{\ell}$, for all $\ell=1, \ldots, L$. These prices are fees to be paid by the customers of class $i$ per unit of time usage.

A customer from class $i$ accepts the posted price $u_{i \ell}$ with probability $p_{i \ell}$, for $l=1,2, \ldots, L$, where these probabilities are assumed to follow Assumption 2.

Assumption 2. (i) $p_{i 1} \geq p_{i 2} \geq \ldots \geq p_{i L}$ is a monotone sequence for $i=1, \ldots, M$; (ii) $u_{i L}$ is defined such that $p_{i L}=0$ for $i=1, \ldots, M$.

Part (i) implies that a customer's willingness to accept an offered price decreases as the price increases. Part (ii) implies that the maximum price, $u_{i L}$, is sufficiently large so that it is always rejected. The SP can reject an arriving customer by posting this reject price.

The total connection fee of a customer depends on the price he accepts and the length of his connection time. We let $\gamma$ denote the continuous-time discount rate, where $\gamma>0$. The expected discounted revenue from a class $i$ customer that accepts the offered price $u_{i \ell}$ is $w_{i \ell}=u_{i \ell} /\left(\mu_{i}+\gamma\right)$, where $w_{i 1}<\cdots<w_{i L}$. When a class $i$ customer accepts the posted price 
Batur et al.: Dynamic Pricing of Wireless Internet

Article submitted to Manufacturing $\&$ Service Operations Management; manuscript no. (Please, provide the manuscript number!) 9

$u_{i \ell}$, the present value of his expected total connection fee at the time of his connection is $w_{i \ell}$. In the remainder of the paper, we will refer to $w_{i \ell}$ as the discounted price.

There may be times when it is necessary to drop a customer who is currently receiving service. In that case, the realized service time will no longer follow an exponential distribution, which significantly complicates the analysis. To ensure tractability, we adopt the following convention: The service times for customer class $i$ follow an exponential $\left(\mu_{i}\right)$ distribution. When a customer of class $i$ arrives and the SP posts the price, the actual service time is unobserved. However, after deciding to accept the price, and before entering into service, the customer realizes her service time, reports this to the SP, and makes the appropriate payment. Thus, we assume that the customer pays upon admission. If the customer is later dropped because a channel becomes unavailable, the SP may refund part of this payment or offer a fixed penalty fee (discussed further below) to the customer.

3.1.2. Channel Classes There are $D$ channel classes, where the classes differ in their degree of availability to the SP. The number of channels of class $d$ available to be used by the SP at time $t$ is denoted by $R_{d}(t)$, where $R_{d}(t) \leq R_{\max , d}$, with $\mathbf{R}(t)=\left(R_{1}(t), \ldots, R_{D}(t)\right)$ and $R(t)=\sum_{d=1}^{D} R_{d}(t)$. We refer to $\mathbf{R}(t)$ or $R(t)$ as the capacity. We will drop the argument $t$ when it is not required for clarity. We use $R_{\max , d}$ to denote the maximum number of channels of class $d$ that can ever be available to the SP, and we define $R_{\max }=\sum_{d=1}^{D} R_{\max , d}$. We refer to $R_{\max }$ as the maximum capacity. As described in Section $1, R_{d}(t)$ is a stochastic process that increases (decreases) in increments of one unit as channels become available (unavailable). Once a channel from class $d$ becomes available, it remains available for a period of time that follows an exponential distribution with rate $\omega_{d}$, and then it becomes unavailable for a period of time that is exponentially distributed with rate $\phi_{d}$. We define $\boldsymbol{\omega}=\left(\omega_{1}, \ldots, \omega_{D}\right)$ and $\boldsymbol{\phi}=\left(\phi_{1}, \ldots, \phi_{D}\right)$.

Since the number of available channels can decrease at any time, it is possible that the total required capacity, i.e., $\mathbf{N}(t)^{\prime} \mathbf{r}$, where $\mathbf{N}(t)^{\prime}$ denotes the transpose of $\mathbf{N}(t)$, may at some time exceed the capacity, $R(t)$. In that case, some customers currently in service will need to be dropped, i.e., have their service immediately terminated. In this case, we assume the SP incurs a fixed drop-off fee, denoted by $K$, which satisfies Assumption 3 .

Assumption 3. The drop-off fee $K$ satisfies $w_{i 1}<\cdots<w_{i, L-1}<K$ for $i=1, \ldots, M$.

The fixed fee, $K$, incurred by the SP may include a penalty fee or partial refund paid to the customer, and/or a goodwill cost incurred by the SP due to customer dissatisfaction. 
Batur et al.: Dynamic Pricing of Wireless Internet
10Article submitted to Manufacturing \& Service Operations Management; manuscript no. (Please, provide the manuscript number!)

3.1.3. System State The state of the system at time $t$, denoted by $\mathbf{S}(t)$, is given by the number of customers of each class currently in the system, as well as the number of available channels from each class, i.e., $\mathbf{S}(t)=(\mathbf{N}(t), \mathbf{R}(t))$. An incoming class $i$ customer can be accepted only if $\mathbf{N}(t)^{\prime} \mathbf{r}+r_{i} \leq R(t)$. Otherwise, that customer must be rejected by the SP by posting the reject price, $u_{i L}$. Hence, the state space is $\mathcal{S}=\left\{(\mathbf{N}, \mathbf{R}): \mathbf{N}^{\prime} \mathbf{r} \leq\right.$ $\left.R, R_{d} \leq R_{\max , d}, d=1, \ldots, D\right\}$.

\subsection{Model Formulation}

We study a continuous-time model with exponential inter-transition times. Thus, the embedded stochastic process is a continuous-time Markov chain. The state of the system changes at each arrival and departure (of customers and channels). The SP must choose the price to post whenever an arrival occurs, and which customer(s) to drop when capacity decreases, i.e., a channel becomes unavailable, and the remaining capacity is insufficient to handle the customers currently in the system. At these decision epochs, the decisions are based on the state of the system, $\mathbf{S}(t)$. This decision process is an infinite horizon, continuous-time Markov decision process (CTMDP). See Puterman (1994) for details.

By using uniformization, we build the discrete-time equivalent of the system (Lippman 1975). The maximum possible rate out of any state is $\Gamma=\sum_{i=1}^{M} \lambda_{i}+\sum_{i=1}^{M} \mu_{i}\left\lfloor\frac{R_{\max }}{r_{i}}\right\rfloor+$ $\sum_{d=1}^{D}\left(\omega_{d}+\phi_{d}\right) R_{\text {max }, d}+\gamma$. Without loss of generality, we assume that $\Gamma$ is 1 .

The value function, $v(\mathbf{N}, \mathbf{R})$, is the total expected discounted stream of future rewards and penalties given the system is in state $(\mathbf{N}, \mathbf{R})$ and is operated optimally. The optimality equation is as follows.

$$
\begin{aligned}
v(\mathbf{N}, \mathbf{R})= & \sum_{i=1}^{M} \lambda_{i} H_{i}(v(\mathbf{N}, \mathbf{R}))+\sum_{i=1}^{M} \mu_{i} N_{i} v\left(\mathbf{N}-\mathbf{e}_{i}^{M}, \mathbf{R}\right) \\
& +\sum_{d=1}^{D} \phi_{d}\left(R_{\max , d}-R_{d}\right) v\left(\mathbf{N}, \mathbf{R}+\mathbf{e}_{d}^{D}\right)+\sum_{d=1}^{D} \omega_{d} R_{d} G_{d}(v(\mathbf{N}, \mathbf{R})) \\
& +\left[\sum_{i=1}^{M} \mu_{i}\left(\left\lfloor\frac{R_{\max }}{r_{i}}\right\rfloor-N_{i}\right)+\sum_{d=1}^{D}\left(\phi_{d} R_{d}+\omega_{d}\left(R_{\max , d}-R_{d}\right)\right)\right] v(\mathbf{N}, \mathbf{R}),
\end{aligned}
$$

where $\mathbf{e}_{i}^{M}\left(\mathbf{e}_{d}^{D}\right)$ is an $M$-dimensional (D-dimensional) unit vector with a 1 in the $i$ th spot ( $d$ th spot) and 0 in the remaining spots. 
Batur et al.: Dynamic Pricing of Wireless Internet

Article submitted to Manufacturing $\mathscr{E}$ Service Operations Management; manuscript no. (Please, provide the manuscript number!) 11

The function $H_{i}(v(\mathbf{N}, \mathbf{R}))$ represents the SP's pricing decision when a class $i$ customer arrives while the system is in state $(\mathbf{N}, \mathbf{R})$. We write $H_{i}$ as follows.

$$
H_{i}(v(\mathbf{N}, \mathbf{R}))= \begin{cases}\max _{\ell}\left[p_{i \ell}\left(v\left(\mathbf{N}+\mathbf{e}_{i}^{M}, \mathbf{R}\right)+w_{i \ell}\right)+\left(1-p_{i \ell}\right) v(\mathbf{N}, \mathbf{R})\right] & \text { if } \mathbf{N}^{\prime} \mathbf{r}+r_{i} \leq R, \\ v(\mathbf{N}, \mathbf{R}) & \text { if } \mathbf{N}^{\prime} \mathbf{r}+r_{i}>R .\end{cases}
$$

The pricing decisions are made only at arrival events with rate $\lambda_{i}$. Hence, rather than tracking revenues in continuous time, we track expected discounted revenues upon arrival.

The second term in the definition of $v(\mathbf{N}, \mathbf{R})$ represents transitions due to customer departures, where class $i$ customers depart at rate $\mu_{i} N_{i}$ when there are $N_{i}$ customers of class $i$ currently being served. The third term in the definition of $v(\mathbf{N}, \mathbf{R})$ represents transitions due to channels becoming available, where class $d$ channels become available at rate $\phi_{d}\left(R_{\max , d}-R_{d}\right)$ when there are $R_{d}$ class $d$ channels currently in use.

In the fourth term in the definition of $v(\mathbf{N}, \mathbf{R})$, class $d$ channels become unavailable at rate $\omega_{d} R_{d}$. The function $G_{d}(v(\mathbf{N}, \mathbf{R}))$ represents the SP's drop-off decision when a class $d$ channel becomes unavailable while the system is in state $(\mathbf{N}, \mathbf{R})$. We write $G_{d}$ as follows.

$$
G_{d}(v(\mathbf{N}, \mathbf{R}))= \begin{cases}\max _{\hat{\mathbf{N}}}\left[v\left(\hat{\mathbf{N}}, \mathbf{R}-\mathbf{e}_{d}^{D}\right)-K \sum_{i=1}^{M}\left(N_{i}-\hat{N}_{i}\right)\right] & \text { if } \mathbf{N}^{\prime} \mathbf{r}>R-1, \\ v\left(\mathbf{N}, \mathbf{R}-\mathbf{e}_{d}^{D}\right) & \text { if } \mathbf{N}^{\prime} \mathbf{r} \leq R-1,\end{cases}
$$

where $\hat{\mathbf{N}}=\left(\hat{N}_{1}, \ldots, \hat{N}_{M}\right), \hat{\mathbf{N}}^{\prime} \mathbf{r} \leq R-1$, and $\hat{N}_{i} \leq N_{i}, i=1,2, \ldots, M$.

Since the drop-off fee, $K$, used in the $G_{d}$ function is fixed per customer, we might intuitively expect that customers requiring the highest bandwidth would be dropped first. Hence, in this paper, we will also study a bandwidth-based drop-off function, denoted by $G_{d}^{B}$, which drops customers according to their bandwidth requirements, i.e., class 1 customers are dropped first, followed by class 2 customers, etc., until the capacity is sufficient to satisfy the bandwidth requirements of the remaining customers. In the following section, we compare the optimal and bandwidth-based drop-off policies in detail. We find that, in most cases, it is optimal to drop the customers with the highest bandwidth requirements first. However, in some cases, it is optimal to drop a customer requiring a lower bandwidth if that customer's expected service time is relatively long (see Theorem 3 below).

The final term in $v(\mathbf{N}, \mathbf{R})$ represents fictitious transitions to the current state $(\mathbf{N}, \mathbf{R})$. The value iteration operator, $T$, is defined as follows, where $f$ is any arbitrary function:

$$
T f(\mathbf{N}, \mathbf{R})=\sum_{i=1}^{M} \lambda_{i} H_{i}(f(\mathbf{N}, \mathbf{R}))+\sum_{i=1}^{M} \mu_{i} N_{i} f\left(\mathbf{N}-\mathbf{e}_{i}^{M}, \mathbf{R}\right)
$$


12Aticing of Wireless Internet 12 Article submitted to Manufacturing $\&$ Service Operations Management; manuscript no. (Please, provide the manuscript number!)

$$
\begin{aligned}
& +\sum_{d=1}^{D} \phi_{d}\left(R_{\max , d}-R_{d}\right) f\left(\mathbf{N}, \mathbf{R}+\mathbf{e}_{d}^{D}\right)+\sum_{d=1}^{D} \omega_{d} R_{d} G_{d}(f(\mathbf{N}, \mathbf{R})) \\
& +\left[\sum_{i=1}^{M} \mu_{i}\left(\left\lfloor\frac{R_{\max }}{r_{i}}\right\rfloor-N_{i}\right)+\sum_{d=1}^{D}\left(\phi_{d} R_{d}+\omega_{d}\left(R_{\max , d}-R_{d}\right)\right)\right] f(\mathbf{N}, \mathbf{R}) .
\end{aligned}
$$

Since $T$ is a contraction mapping, it can be repeatedly applied to find the value function through successive approximation. If $v_{0}$ represents an initial estimate of $v$, then one pass of the value iteration algorithm (VIA) produces $v_{1}=T v_{0}$, and $q$ applications of $T$ produces $v_{q}=T v_{q-1}=T^{q} v_{0}$. Since the state space, $\mathcal{S}$, and the one-period payments are finite, there exists a unique $v$ such that $T v=v$ and $\lim _{q \rightarrow \infty} v_{q}=v$. See Theorem 6.2.5 in Puterman (1994). Hence, VIA can be used to find the $v$ values. See Section 6.3 in Puterman (1994). The rewards and transition probabilities are stationary. Since the state space, $\mathcal{S}$, the action space, $\mathcal{U}_{i}$ for $i=1, \ldots, M$, and the one-period payments are finite, there exists an optimal deterministic stationary policy. See Theorem 6.2.10 in Puterman (1994).

\subsection{Dynamic Pricing Policy}

The optimal dynamic pricing policy for the SP is found by solving the optimization in the $H_{i}(v(\mathbf{N}, \mathbf{R}))$ function, which determines the optimal price to offer a class $i$ customer, given the state of the system. To characterize this pricing policy, define $A_{i}(\mathbf{N}, \mathbf{R})$ to be the opportunity cost associated with the acceptance of an arriving class $i$ customer when the system is in state $(\mathbf{N}, \mathbf{R})$, where $A_{i}(\mathbf{N}, \mathbf{R})=v(\mathbf{N}, \mathbf{R})-v\left(\mathbf{N}+\mathbf{e}_{i}^{M}, \mathbf{R}\right) \geq 0$. As in Gans and Savin (2007), we let $g_{i}\left(w_{i \ell} \mid A_{i}(\mathbf{N}, \mathbf{R})\right)=p_{i \ell}\left(-A_{i}(\mathbf{N}, \mathbf{R})+w_{i \ell}\right)$ denote the expected net gain from offering the discounted price $w_{i \ell}$ to a class $i$ customer, given the state $(\mathbf{N}, \mathbf{R})$; and let $g_{i}^{*}(\mathbf{N}, \mathbf{R}) \equiv g_{i}^{*}\left(A_{i}(\mathbf{N}, \mathbf{R})\right)$ denote the expected net gain from offering the optimal discounted price to a class $i$ customer. In other words, $g_{i}^{*}\left(A_{i}(\mathbf{N}, \mathbf{R})\right)=$ $\max _{w_{i \ell}} g_{i}\left(w_{i \ell} \mid A_{i}(\mathbf{N}, \mathbf{R})\right)=\max _{\ell}\left[p_{i \ell}\left(-A_{i}(\mathbf{N}, \mathbf{R})+w_{i \ell}\right)\right]$. Note that finding $g_{i}^{*}\left(A_{i}(\mathbf{N}, \mathbf{R})\right)$ is equivalent to the optimization in $H_{i}(v(\mathbf{N}, \mathbf{R}))$. Finally, let $w_{i}^{*}(\mathbf{N}, \mathbf{R}) \equiv w_{i \ell_{i}^{*}\left(A_{i}(\mathbf{N}, \mathbf{R})\right)}$, where $\ell_{i}^{*}\left(A_{i}(\mathbf{N}, \mathbf{R})\right)=\arg \max _{\ell}\left[p_{i \ell}\left(-A_{i}(\mathbf{N}, \mathbf{R})+w_{i \ell}\right)\right]$, denote the optimal discounted price to offer a class $i$ customer who arrives while the system is in state $(\mathbf{N}, \mathbf{R})$. The optimal discounted prices are dependent on the state, $(\mathbf{N}, \mathbf{R})$, only through the opportunity cost, $A_{i}$. Therefore, for notational simplicity, we suppress the $(\mathbf{N}, \mathbf{R})$ notation when it is not required for clarity, i.e., we write $g_{i}^{*}\left(A_{i}\right)$ and $\ell_{i}^{*}\left(A_{i}\right)$. We can now state the following result. 
Batur et al.: Dynamic Pricing of Wireless Internet

Article submitted to Manufacturing $\mathscr{E}$ Service Operations Management; manuscript no. (Please, provide the manuscript number!) 13

THEOREM 1. The expected net gain from offering the optimal price to an arriving class

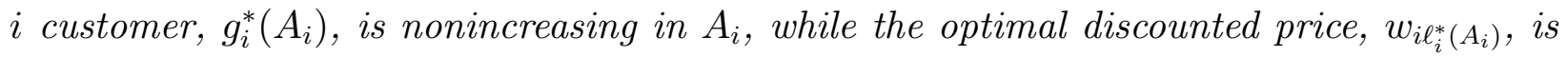
nondecreasing in $A_{i}$.

Thus, to prove structural properties for the optimal dynamic pricing policy, it is sufficient to understand the behavior of $A_{i}$, the opportunity cost associated with the acceptance of an arriving class $i$ customer. The proof of Theorem 1 is given in Appendix A.

When $A_{i}=0$, let $m_{i}=\arg \max _{\ell}\left(p_{i \ell} w_{i \ell}\right)$. The price $w_{i m_{i}}$ is the myopic price, i.e., the price that maximizes the immediate expected revenue from a class $i$ customer. Since the optimal discounted price, $w_{i \ell_{i}^{*}\left(A_{i}\right)}$, is nondecreasing in $A_{i}$, the myopic price is a lower bound on the optimal price in any state.

Corollary 1. The optimal discounted prices satisfy $w_{i}^{*}(\mathbf{N}, \mathbf{R}) \geq w_{i_{i}}$ for all $(\mathbf{N}, \mathbf{R})$.

Theorem 1 and Corollary 1 together imply that if the condition

$$
p_{i m_{i}}\left(-A_{i}+w_{i m_{i}}\right) \geq p_{i \ell}\left(-A_{i}+w_{i \ell}\right) \text { for all } \ell=m_{i}+1, \ldots, L,
$$

is satisfied, then $w_{i m_{i}}$ is the optimal discounted price to offer to a class $i$ customer. Equation (7) implies that if

$$
A_{i} \leq\left(p_{i m_{i}} w_{i m_{i}}-p_{i l} w_{i \ell}\right) /\left(p_{i m_{i}}-p_{i \ell}\right)
$$

for all $\ell=m_{i}+1, \ldots, L$, then $w_{i m_{i}}$ is the optimal discounted price to offer to a class $i$ customer. Hence, when the opportunity cost associated with the acceptance of an arriving customer is sufficiently small, the myopic price is the optimal price. However, if the opportunity cost is larger than a threshold, the optimal price will be greater than the myopic price. Similar results are proven in the literature for various revenue management problems. See, for example, Bitran and Caldentey (2003).

\section{Structural Properties of the MDP}

Next, for several different problem settings, we analyze the MDP formulation in order to derive insights into the structure of the SP's optimal dynamic pricing policy.

\section{1. $\quad M$ Customer Classes and $D$ Channel Classes}

For the general setting, the total expected discounted stream of future rewards and penalties decreases as we have more customers in service, and increases as we have more channel availability. In other words, more congestion leads to lower expected profit. Also, for a 
Batur et al.: Dynamic Pricing of Wireless Internet
14Article submitted to Manufacturing \& Service Operations Management; manuscript no. (Please, provide the manuscript number!) given set of parameter values, if we only increase the arrival rate to the system, this larger demand leads to higher expected profit. Specifically, we have the following theorem:

THEOREM 2. The optimal value function $v(\mathbf{N}, \mathbf{R})$ has the following properties:

1. $v(\mathbf{N}, \mathbf{R})$ is nonincreasing in $N_{j}$, i.e., $v\left(\mathbf{N}+\mathbf{e}_{j}^{M}, \mathbf{R}\right) \leq v(\mathbf{N}, \mathbf{R})$, for $j=1, \ldots, M$.

2. $v(\mathbf{N}, \mathbf{R})$ is nondecreasing in $R_{d}$, i.e., $v\left(\mathbf{N}, \mathbf{R}+\mathbf{e}_{d}^{D}\right) \geq v(\mathbf{N}, \mathbf{R})$, for $d=1, \ldots, D$.

3. $v(\mathbf{N}, \mathbf{R})$ is a nondecreasing function of the arrival rates, $\lambda_{j}, j=1, \ldots, M$.

The proof of Theorem 2 is given in Appendix B. We next show how the different service rates and remaining capacity in a given state impact the value function.

TheOREM 3. Assume that $\mu_{j} \leq \mu_{k}$ for all $j, k=1, \ldots, M$, and $j<k$, then

$$
v\left(\mathbf{N}+\mathbf{e}_{j}^{M}, \mathbf{R}\right) \leq v\left(\mathbf{N}+\mathbf{e}_{k}^{M}, \mathbf{R}\right)
$$

The proof of Theorem 3 is given in Appendix C. When $j<k$, Assumption 1 implies $r_{j} \geq r_{k}$. Hence, the remaining (i.e., unused) capacity is smaller in state $\left(\mathbf{N}+\mathbf{e}_{j}^{M}, \mathbf{R}\right)$ than in state $\left(\mathbf{N}+\mathbf{e}_{k}^{M}, \mathbf{R}\right)$. According to Theorem 3, when $\mu_{j} \leq \mu_{k}$, admitting a class $k$ customer, rather than a class $j$ customer, takes the system to a state with relatively larger value due to the larger remaining capacity in state $\left(\mathbf{N}+\mathbf{e}_{k}^{M}, \mathbf{R}\right)$. However, when $\mu_{j}>\mu_{k}$, the opposite might hold, i.e., it might be preferable to admit a class $j$ customer with higher bandwidth requirement, rather than a class $k$ customer with smaller bandwidth requirement. This is due to the fact that, when $\mu_{j}>\mu_{k}$, the expected service time of a class $j$ customer is smaller than that of a class $k$ customer, i.e., $1 / \mu_{j}<1 / \mu_{k}$.

This theorem has implications for the optimal drop-off policy, represented by the $G_{d}$ function in Equation (5). In most cases, when there is a shortage of resources, i.e., when $R>$ $\mathbf{N}^{\prime} \mathbf{r}$, it is optimal to drop the customer(s) with the highest bandwidth requirement, which we refer to as the bandwidth-based drop-off policy. However, when customers requiring lower bandwidth have longer expected service times than customers requiring higher bandwidth, the bandwidth-based drop-off policy may not be optimal. Thus, when the turn-over rate of low bandwidth customers is small, i.e., they stay in the system for a long time, it may be optimal to drop those low-bandwidth customers first.

In the light of this theorem, we also considered an alternative heuristic drop-off policy which drops customers based on the magnitude of $r_{i} / \mu_{i}$, i.e., customers with the largest value of $r_{i} / \mu_{i}$ are dropped first. Our experimental results indicate that this alternative 
Batur et al.: Dynamic Pricing of Wireless Internet

Article submitted to Manufacturing $\&$ Service Operations Management; manuscript no. (Please, provide the manuscript number!) 15

drop-off policy does not perform considerably better than the proposed bandwidth-based drop-off policy.

The following theorem, whose proof is in Appendix D, demonstrates that the bandwidthbased drop-off policy and the optimal drop-off policy always drop the same number of customers. However, the classes of the dropped customers may not be the same.

THEOREM 4. The total number of customers that will be dropped according to the optimal drop-off function $G_{d}$ in Equation (5) and the bandwidth-based drop-off function $G_{d}^{B}$ are exactly the same.

In the general case of $M$ customer classes and $D$ channel classes, structural properties for the value function, such as concavity and submodularity, are not provable. Even in the special case of $M=2$ customer classes and $D$ channel classes, the concavity of the optimal value function in $N_{1}$ and $N_{2}$ is not provable. A similar observation can be made about Gans and Savin (2007) and Ulukus et al. (2011), i.e., these authors are also not able to prove the concavity of the value function when there are two customer classes with different service rates. Because we are unable to prove concavity, we cannot prove that the optimal price, $w_{i}^{*}\left(N_{1}, N_{2}, \mathbf{R}\right)$, is nondecreasing in $N_{i}, i=1,2$. In fact, experimental results presented in Section 6 indicate that, in some states, the optimal prices for class $i$, $w_{i}^{*}\left(N_{1}, N_{2}, \mathbf{R}\right)$, are decreasing in $N_{i}$, a result that may seem counter-intuitive.

Similarly, when $M=2$, submodularity of the optimal value function in $N_{1}$ and $N_{2}$ is not provable, and thus we cannot prove that the optimal discounted price for an arriving class $i$ customer, $w_{i}^{*}\left(N_{1}, N_{2}, \mathbf{R}\right)$, is nondecreasing in $N_{j}, i, j=1,2, i \neq j$. However, if we use the bandwidth-based drop-off function, $G_{d}^{B}$, we can show that the optimal value function is submodular in $N_{1}$ and $N_{2}$, and thus $w_{i}^{*}\left(N_{1}, N_{2}, \mathbf{R}\right)$ is nondecreasing in $N_{j}, i, j=1,2, i \neq j$. In other words, the price offered to an arriving class $i$ customer will generally be increasing in the number of class $j$ customers already in the system. The fact that submodularity of the value function is dependent on the use of the bandwidth-based drop-off policy can be explained by Theorem 3. Under the optimal drop-off policy, it is sometimes optimal to drop the low-bandwidth customer(s) of class 2 first, even when there are high-bandwidth class 1 customers in the system. However, under the bandwidth-based drop-off policy, class 1 customers are always dropped first. 
Batur et al.: Dynamic Pricing of Wireless Internet 16Article submitted to Manufacturing \& Service Operations Management; manuscript no. (Please, provide the manuscript number!)

\subsection{Similar Customer Classes}

We next consider the case in which the customer classes are similar, i.e., have a common service rate and bandwidth requirement, $\mu_{1}=\ldots=\mu_{M}=\mu$ and $r_{1}=\ldots=r_{M}=r$, but their arrival rates and price sensitivities may differ. In this case, we do not need to distinguish between the customer classes once they are admitted, which reduces the size of the state space. Since the bandwidth requirements are the same for all classes, the price sets will be the same, i.e., $\mathcal{U}_{1}=\ldots=\mathcal{U}_{M}=\left\{r u_{1}, \ldots, r u_{L}\right\}$. Since the service rates are identical, we have $w_{1 \ell}=\ldots=w_{M \ell}=w_{\ell}, \ell=1, \ldots, L$.

4.2.1. $\quad M$ Similar Customer Classes and $D$ Channel Classes In the setting with a general number of customer classes and channel classes, the state is $(N, \mathbf{R})$, where $N$ is the total number of customers that are currently in the system. From Theorems 3 and 4, we can conclude that the bandwidth-based drop-off policy is optimal in this case. The formulation of the bandwidth-based drop-off function in this case is as follows.

$$
G_{d}^{B}(v(N, \mathbf{R}))= \begin{cases}v\left(\left\lfloor\frac{R-1}{r}\right\rfloor, \mathbf{R}-\mathbf{e}_{d}^{D}\right)-K\left(N-\left\lfloor\frac{R-1}{r}\right\rfloor\right) & \text { if } N r>R-1, \\ v\left(N, \mathbf{R}-\mathbf{e}_{d}^{D}\right) & \text { if } N r \leq R-1\end{cases}
$$

for $d=1, \ldots, D$. Since it is easier to prove results using the bandwidth-based drop-off function formulation, $G_{d}^{B}$ will be used in all of the proofs in this section.

Given this problem formulation, we can derive some important properties for the value function, which are useful for characterizing the optimal dynamic pricing policy. The properties proven in Theorem 2 apply to the value function $v(N, \mathbf{R})$ as well.

THEOREM 5. The optimal value function $v(N, \mathbf{R})$ has the following properties:

1. $v(N, \mathbf{R})$ is concave in $N$, i.e., $v(N+2, \mathbf{R})-v(N+1, \mathbf{R}) \leq v(N+1, \mathbf{R})-v(N, \mathbf{R})$.

2. $v(N, \mathbf{R})$ is supermodular on $\mathcal{S}$, i.e., $v\left(N+1, \mathbf{R}+\mathbf{e}_{d}^{D}\right)-v(N+1, \mathbf{R}) \geq v\left(N, \mathbf{R}+\mathbf{e}_{d}^{D}\right)-$ $v(N, \mathbf{R})$.

The proof of Theorem 5 is analogous to the proof of Theorem 2 . Notice that, when the customer classes are similar, we are able to prove the concavity of the value function. In the literature, it is not uncommon for the value function to be concave in the number of customers in the system when the service characteristics of all customer classes are the same (in our case, the service rates and bandwidth requirements are the same). See, for example, Miller (1969) and Gans and Savin (2007). 
Batur et al.: Dynamic Pricing of Wireless Internet

Article submitted to Manufacturing $\mathscr{E}$ Service Operations Management; manuscript no. (Please, provide the manuscript number!) 17

The supermodularity result in Theorem 5 implies that the value of having an additional channel of any class is increasing in the number of customers in the system. This property is different from monotonicity of the value function with respect to the number of servers, a result that is typically proven in previous literature, e.g., Aktaran-Kalayci et al. (2009). While monotonicity implies that the value increases as the number of servers increases, supermodularity considers how that rate of increase in the value function depends on the number of customers in the system. Further, when the previous literature considers how a change in the number of servers affects the value function, the change in the number of servers is permanent (i.e., the literature compares a setting in which the system has $s$ servers to a setting in which the system has $s+1$ servers). In contrast, in our setting, we are considering how the value function changes with a change in the system state (i.e., the available channels $\mathbf{R}$ ), which is a temporary change (i.e., the number of channels fluctuates over time as channels become available and unavailable).

We next discuss the optimal dynamic pricing policy. We use $A(N, \mathbf{R})$ to represent the opportunity cost associated with the acceptance of an arriving customer when the system is in state $(N, \mathbf{R})$, where $A(N, \mathbf{R})=v(N, \mathbf{R})-v(N+1, \mathbf{R}) \geq 0$. We drop the subscript on $A_{i}$ since we do not need to differentiate by customer class. From Theorem 5 , we know that $v$ is concave in $N$ and supermodular in $N$ and $\mathbf{R}$. Hence, $A(N, \mathbf{R})$ is nondecreasing in $N$ and nonincreasing in $R_{d}$. Therefore, using Theorem 1, we can state the following result:

COROLLARY 2. The expected net gain from offering the optimal price to an arriving class $i$ customer, $g_{i}^{*}(N, \mathbf{R})$, is nonincreasing in $N$ and nondecreasing in $R_{d}$, while the optimal discounted prices are nondecreasing in $N$ and nonincreasing in $R_{d}$, i.e.,

$$
w_{i}^{*}(N, \mathbf{R}) \leq w_{i}^{*}(N+1, \mathbf{R}) \quad \text { and } \quad w_{i}^{*}(N, \mathbf{R}) \geq w_{i}^{*}\left(N, \mathbf{R}+\mathbf{e}_{d}^{D}\right) .
$$

Similar relationships hold for $u_{i}^{*}(N, \mathbf{R})$.

Thus, the optimal prices are increasing in the number of customers in the system, $N$, and decreasing in the number of available channels, $R=\sum_{d=1}^{D} R_{d}$. Previous literature, e.g., Aktaran-Kalayci et al. (2009), has also found that the optimal prices are decreasing in the number of servers and in the capacity of the system. However, in that literature, the number of servers and capacity are exogenously specified parameters. In contrast, in our setting, the number of available channels is part of the system state and varies stochastically. 
Overall, we have that the prices are decreasing in the remaining capacity, which can be measured by $R-N$. Using Corollary 1 and Equation (8), we can also observe that myopic pricing will be optimal when the remaining capacity is sufficiently large.

We next present some sensitivity analysis results for the optimal pricing policy.

THEOREM 6. The optimal pricing policy has the following properties:

1. $w_{i}^{*}(N, \mathbf{R})$ and $u_{i}^{*}(N, \mathbf{R})$ are nondecreasing functions of the arrival rates, $\lambda_{j}, j=$ $1, \ldots, M$.

2. $w_{i}^{*}(N, \mathbf{R})$ is a nonincreasing function of the service rate, $\mu$.

3. $w_{i}^{*}(N, \mathbf{R})$ and $u_{i}^{*}(N, \mathbf{R})$ are nondecreasing functions of the drop-off fee, $K$.

The proof of Theorem 6 is given in Appendix E. Theorem 6.1 indicates that an increase in the arrival rate for any given customer class will cause an increase in optimal prices of all classes. Intuitively, an increase in the arrival rate for any class will increase the congestion in the system, requiring higher prices. Theorem 6.2 indicates that when customers can be cleared from the system more quickly, optimal prices can be reduced. Notice that the relationship presented in Theorem 6,2 holds only for $w_{i}^{*}(N, \mathbf{R})$, and is not valid for $u_{i}^{*}(N, \mathbf{R})$. Recall that $u_{i}^{*}=w_{i}^{*}(\mu+\gamma)$. When $\mu$ increases and $w_{i}^{*}$ decreases, the behavior of $u_{i}^{*}$ is unclear. Theorem 6.3 indicates that an increase in the drop-off fee, $K$, increases the optimal prices for all customer classes when the customer classes are similar.

Similar results are common in the literature on pricing of queueing systems. For example, Aktaran-Kalayci et al. (2009) provide similar monotonicity results for the optimal prices with respect to the arrival and service rates for an $M / M / s / K$ system with a single customer class. In addition, for a large class of single-dimension queueing systems, Cil et al. (2009) discuss structural properties of the value function (and, specifically, for the opportunity cost, which is analogous to $A_{i}$, as defined above) that are sufficient to ensure that the optimal prices will have similar monotonicity properties with respect to the arrival and service rates. However, it is important to note that none of the systems studied in these previous works considers capacity that evolves as a stochastic process, with the potential for customer drop-offs. Thus, these previous works also do not present analogous results regarding the monotonicity of the prices with respect to the drop-off fee.

We next consider the impact of customer's price sensitivity on the optimal prices. 
Batur et al.: Dynamic Pricing of Wireless Internet

Article submitted to Manufacturing $\&$ Service Operations Management; manuscript no. (Please, provide the manuscript number!) 19

TheOREm 7. For classes $j$ and $k$, if $p_{j \ell} / p_{k \ell}$ is an increasing function of $\ell$ for $\ell=$ $1, \ldots, L-1$, then $w_{j}^{*}(N, \mathbf{R}) \geq w_{k}^{*}(N, \mathbf{R})$ for all $(N, \mathbf{R})$. A similar result holds for $u_{j}^{*}(N, \mathbf{R})$ and $u_{k}^{*}(N, \mathbf{R})$.

The proof of Theorem 7 is given in Appendix F. The theorem implies that customers who are more price sensitive will be offered lower prices. Gans and Savin (2007) prove a similar result for walk-in customers. The condition in Theorem 7, i.e., $p_{j l} / p_{k l}$ is an increasing function of $l$, implies that class $k$ customers are more price sensitive than class $j$ customers. Recall that $p_{i l}$ denotes the probability that a class $i$ customer will accept the posted price $r u_{l}$, where the prices are ordered such that $u_{1}<u_{2}<\cdots<u_{L}$. Assumption 2 implies that $p_{i 1} \geq p_{i 2} \geq \cdots \geq p_{i L}$ for all $i$. Thus, for all customer classes, the probability of accepting a posted price decreases as the price increases. Hence, in the ratio $p_{j l} / p_{k l}$, both $p_{j l}$ and $p_{k l}$ are decreasing in $l$. The condition that $p_{j l} / p_{k l}$ is an increasing function of $l$ implies that $p_{k l}$ decreases more rapidly in $l$ than does $p_{j l}$, which implies that class $k$ customers are more price sensitive than class $j$ customers.

4.2.2. $M$ Similar Customer Classes and Two Channel Classes In this section, we consider a setting with $M$ similar customer classes and just $D=2$ channel classes in order to consider how the relative magnitudes of the channel availability $\left(\omega_{d}\right)$ and unavailability $\left(\phi_{d}\right)$ rates affect the optimal prices. We present two theorems to demonstrate the results.

THEOREM 8. Suppose the two channel classes have the same availability rate and maximum capacity, i.e., $\omega_{1}=\omega_{2}=\omega$ and $R_{\max , 1}=R_{\max , 2}=0.5 R_{\max }$. However, the unavailability rates may differ, i.e., $\phi_{1} \geq \phi_{2}$. Then the optimal pricing policy satisfies $w_{i}^{*}\left(N, R_{1}+1, R_{2}\right) \geq$ $w_{i}^{*}\left(N, R_{1}, R_{2}+1\right)$. A similar relationship holds for $u_{i}^{*}\left(N, R_{1}, R_{2}\right)$.

The proof of Theorem 8 uses Theorem 1 and is analogous to the proof Theorem 5 . Under the conditions of Theorem 8 , the probability of a channel becoming unavailable in states $\left(N, R_{1}+1, R_{2}\right)$ and $\left(N, R_{1}, R_{2}+1\right)$ are the same and equal to $\left(R_{1}+R_{2}+1\right) \omega$. However, the probability of a channel becoming available in state $\left(N, R_{1}+1, R_{2}\right)$ is $\left(0.5 R_{\max }-R_{1}-\right.$ 1) $\phi_{1}+\left(0.5 R_{\max }-R_{2}\right) \phi_{2}$. This is less than or equal to the probability of a channel becoming available in state $\left(N, R_{1}, R_{2}+1\right)$, which is $\left(0.5 R_{\max }-R_{1}\right) \phi_{1}+\left(0.5 R_{\max }-R_{2}-1\right) \phi_{2}$. Hence, there is relatively less capacity in state $\left(N, R_{1}+1, R_{2}\right)$ than in state $\left(N, R_{1}, R_{2}+1\right)$, which leads to higher optimal discounted prices in state $\left(N, R_{1}+1, R_{2}\right)$. 
Batur et al.: Dynamic Pricing of Wireless Internet
20Article submitted to Manufacturing \& Service Operations Management; manuscript no. (Please, provide the manuscript number!)

THEOREM 9. Suppose the two channel classes have the same unavailability rate and maximum capacity, i.e., $\phi_{1}=\phi_{2}=\phi$ and $R_{\max , 1}=R_{\max , 2}=0.5 R_{\max }$. However, the availability rates may differ, i.e., $\omega_{1} \geq \omega_{2}$. Then the optimal pricing policy satisfies $w_{i}^{*}\left(N, R_{1}+\right.$ $\left.1, R_{2}\right) \geq w_{i}^{*}\left(N, R_{1}, R_{2}+1\right)$. A similar relationship holds for $u_{i}^{*}\left(N, R_{1}, R_{2}\right)$.

The proof of Theorem 9 uses Theorem 1 and is analogous to the proof Theorem 5 . Under the conditions given in Theorem 9 , the probability of a channel becoming available in states $\left(N, R_{1}+1, R_{2}\right)$ and $\left(N, R_{1}, R_{2}+1\right)$ are the same and equal to $\left(0.5 R_{\max }-R_{1}-R_{2}-1\right) \phi$. However, the probability of a channel becoming unavailable in state $\left(N, R_{1}+1, R_{2}\right)$ is $\left(R_{1}+1\right) \omega_{1}+R_{2} \omega_{2}$. This is larger than or equal to the probability of a channel becoming unavailable in state $\left(N, R_{1}, R_{2}+1\right)$, which is $R_{1} \omega_{1}+\left(R_{2}+1\right) \omega_{2}$. Hence, there is relatively less capacity in state $\left(N, R_{1}+1, R_{2}\right)$ than in state $\left(N, R_{1}, R_{2}+1\right)$, which leads to higher optimal discounted prices in state $\left(N, R_{1}+1, R_{2}\right)$.

\section{Heuristic Policies}

The optimal dynamic pricing policy can be determined through the VIA when the state space is reasonably small. However, for realistic problem sizes, solving the VIA is infeasible. Therefore, we propose three heuristics that approximate the optimal dynamic pricing policy by aggregating either the customer classes, the channel classes, or both. In these heuristics, we utilize the VIA in the aggregate state space. Thus, the pricing policy obtained from the heuristic is suboptimal for the original (non-aggregate) state space. In Section 6, we will numerically evaluate the performance of these proposed dynamic pricing heuristics, and compare their performance to both the myopic and optimal static pricing policies.

\section{1. $N$-Heuristic}

In the $N$-heuristic, which is motivated by the optimal policy for the model in Section 4.2.1 with $M$ similar customer classes and $D$ channel classes, we aggregate the customer classes into a single average class. Let $N(t)$ be the number of customers in the system at time $t$, where $N(t)=\sum_{i=1}^{M} N_{i}(t)$. The aggregate state is $\mathbf{S}(t)=\left(N(t), R_{1}(t), \ldots, R_{D}(t)\right)$. Thus, once a customer is admitted to the system, the heuristic does not keep track of the customer's class. Therefore, we model the system using the average bandwidth requirement, $\bar{r}$, and average service rate, $\bar{\mu}$, across the customer classes. However, the heuristic does consider the customer class at the time of a customer arrival to the system, i.e., the price posted to an arriving customer will depend on that customer's price sensitivity (through 
Batur et al.: Dynamic Pricing of Wireless Internet

Article submitted to Manufacturing $\&$ Service Operations Management; manuscript no. (Please, provide the manuscript number!) 21

the $p_{i \ell}$ probabilities). Further, the customer's bandwidth requirement, $r_{i}$, is considered when determining whether or not the customer can be admitted to the system. Thus, an incoming class $i$ customer can be accepted if $N(t) \bar{r}+r_{i} \leq R(t)$, where $R(t)=\sum_{d=1}^{D} R_{d}(t)$, and the state space is $\mathcal{S}=\left\{(N, \mathbf{R}): N \bar{r} \leq R, R_{d} \leq R_{\max , d}, d=1, \ldots, D\right\}$.

The VIA for the $N$-Heuristic can be used to determine a pricing policy. However, since the heuristic aggregates customers once they are admitted to the system, it cannot be used to determine a drop-off policy which considers the classes of the customers that are currently in the system. When the heuristic is implemented, however, it is necessary to have a drop-off policy to determine how to drop customers when the bandwidth requirement exceeds the available capacity. Thus, for the implementation of the $N$-Heuristic, we propose to use the bandwidth-based drop-off policy presented in Section 3.2. As discussed above, due to Theorems 3 and 4 , this policy is known to be optimal under certain conditions.

\section{2. $\quad R$-Heuristic}

In the $R$-heuristic, in contrast to the $N$-heuristic, we aggregate the channel classes into a single average class. We model the system using the average unavailability rate, $\bar{\phi}$, and average availability rate, $\bar{\omega}$, across the classes. The aggregate state is $\mathbf{S}(t)=$ $\left(N_{1}(t), \ldots, N_{M}(t), R(t)\right)$. An incoming class $i$ customer can be accepted if $\mathbf{N}(t)^{\prime} \mathbf{r}+r_{i} \leq R(t)$. Thus, the state space is $\mathcal{S}=\left\{(\mathbf{N}, R): \mathbf{N}^{\prime} \mathbf{r} \leq R, R \leq R_{\max }\right\}$. Unlike the $N$-Heuristic, the $R$-Heuristic provides both a pricing policy and a drop-off policy because the customers' classes can be distinguished while they are in the system. An interesting question, which will be considered in Section 6, is whether we lose more, relative to the optimal policy, by aggregating customer classes (the $N$-heuristic) or channel classes (the $R$-heuristic).

\section{3. $(N, R)$-Heuristic}

In the $(N, R)$-heuristic, we aggregate both the customer classes and the channel classes. We model the system using the average bandwidth requirement, $\bar{r}$; average service rate, $\bar{\mu}$; and the average channel rates, $\bar{\phi}$ and $\bar{\omega}$. The aggregate state is $\mathbf{S}(t)=(N(t), R(t))$. As in the $N$-heuristic, the $(N, R)$-heuristic does consider the customer class at the time of a customer arrival to the system. However, once admitted, the customer's class is no longer tracked. An incoming class $i$ customer can be accepted if $N(t) \bar{r}+r_{i} \leq R(t)$. Thus, the state space is $\mathcal{S}=\left\{(N, R): N \bar{r} \leq R, R \leq R_{\max }\right\}$. Similar to the $N$-heuristic, the $(N, R)$ heuristic provides a pricing policy, but not a drop-off policy. Hence, in implementation of the $(N, R)$-heuristic, we assume that the bandwidth-based drop-off policy will be used. 
The algorithm for the $(N, R)$-heuristic is:

1. Set $v_{0}(N, R)=0$. Specify $\varepsilon>0$, and set $q=0$. Compute the class $i$ price set $\mathcal{U}_{i}=$ $\left\{u_{i 1}, u_{i 2}, \ldots, u_{i L}\right\}$ from $\mathcal{U}=\left\{u_{1}, u_{2}, \ldots, u_{L}\right\}$ by $u_{i \ell}=r_{i} u_{\ell}$ for $\ell=1, \ldots, L$.

2. For each $(N, R) \in \mathcal{S}\left(N \bar{r} \leq R\right.$ and $\left.R \leq R_{\max }\right)$, compute $v_{q+1}(N, R)$ by

$$
\begin{array}{r}
v_{q+1}(N, R)=\left\{\sum_{i=1}^{M} \lambda_{i} H_{i}\left(v_{q}(N, R)\right)+\bar{\mu} N v_{q}(N-1, R)+\bar{\phi}\left(R_{\max }-R\right) v_{q}(N, R+1)\right. \\
\left.+\bar{\omega} R G^{B}\left(v_{q}(N, R)\right)+\left[\bar{\mu}\left(\left\lfloor\frac{R_{\overline{m a x}}}{\bar{r}}\right\rfloor-N\right)+\bar{\phi} R+\bar{\omega}\left(R_{\max }-R\right)\right] v_{q}(N, R)\right\} / \Gamma,
\end{array}
$$

where $\Gamma=\sum_{i=1}^{M} \lambda_{i}+\bar{\mu}\left\lfloor\frac{R_{\max }}{\bar{r}}\right\rfloor+(\bar{\phi}+\bar{\omega}) R_{\max }$;

$$
H_{i}\left(v_{q}(N, R)\right)= \begin{cases}\max _{\ell}\left[p_{i \ell}\left(v_{q}(N+1, R)+w_{i \ell}\right)+\left(1-p_{i \ell}\right) v_{q}(N, R)\right] & \text { if } N \bar{r}+r_{i} \leq R, \\ v_{q}(N, R) & \text { if } N \bar{r}+r_{i}>R,\end{cases}
$$

where $w_{i \ell}=\frac{u_{i \ell}}{\mu_{i}}$ for all $i=1, \ldots, M$ and $\ell=1, \ldots, L$; and

$$
G^{B}\left(v_{q}(N, R)\right)= \begin{cases}v_{q}\left(\left\lfloor\frac{R-1}{\bar{r}}\right\rfloor, R-1\right)-K\left(N-\left\lfloor\frac{R-1}{\bar{r}}\right\rfloor\right) & \text { if } N \bar{r}>R-1 \\ v_{q}(N, R-1) & \text { if } N \bar{r} \leq R-1\end{cases}
$$

3. Set

$$
M_{q}=\max _{(N, R) \in \mathcal{S}}\left(v_{q+1}(N, R)-v_{q}(N, R)\right) \quad \text { and } \quad m_{q}=\min _{(N, R) \in \mathcal{S}}\left(v_{q+1}(N, R)-v_{q}(N, R)\right) .
$$

If $0 \leq \frac{M_{q}-m_{q}}{m_{q}} \leq \varepsilon$, go to step 4. Otherwise increment $q$ by 1 and return to step 2 .

4. For each $(N, R) \in \mathcal{S}$ and $i, i=1, \ldots, M$ :

if $N \bar{r}+r_{i} \leq R$, choose

$$
u_{i}^{\varepsilon}(N, R) \in \arg \max _{\ell}\left[p_{i \ell}\left(v_{q+1}(N+1, R)+w_{i \ell}\right)+\left(1-p_{i \ell}\right) v_{q+1}(N, R)\right]
$$

else write "capacity not available." Stop.

\subsection{Myopic and Optimal Static Pricing Policies}

The above heuristics consider state-dependent (or dynamic) pricing policies. We also consider the performance of two static pricing policies, myopic and optimal static pricing, in which the same price is offered to all customers of a given class, i.e., the price is not dependent on the system state. Under the myopic policy, the SP determines whether there is sufficient capacity to admit the arriving class $i$ customer. If not, the reject price $\left(u_{i L}\right)$ is posted. If so, the SP posts the myopic price, which is the price that maximizes the immediate expected discounted revenue earned from admitting the customer. The myopic policy 
Batur et al.: Dynamic Pricing of Wireless Internet

Article submitted to Manufacturing \& Service Operations Management; manuscript no. (Please, provide the manuscript number!) 23

is a useful benchmark for evaluating the proposed dynamic pricing heuristics because it is easy to compute and to implement, and has been studied in the previous literature.

In addition, we will evaluate the performance of the optimal static pricing policy, in which we find the optimal constant price to charge to each customer class. Unfortunately, finding the optimal static pricing policy is computationally challenging. The previous literature on optimal static pricing policies (e.g., Gayon et al. (2009)) generally considers just a single customer class, which requires optimization over a single dimension. In our setting, however, the static pricing policy must specify a price for each customer class. To find the optimal static pricing policy, we perform an exhaustive search, where the long-run average value of a given pricing policy is evaluated using simulation, as described in Section 6 . When searching for the optimal price for customer class $i$, in order to ensure fair comparison with the dynamic pricing policies, we start with the price set $\mathcal{U}_{i}$, and then we exclude from this set all prices that are less than the myopic price for class $i$. If we let $\mathcal{U}_{i}^{S}$ denote the set of remaining prices for class $i$, then the optimal static pricing policy has search space with size $\left|\mathcal{U}_{1}^{S}\right| \times\left|\mathcal{U}_{2}^{S}\right| \times \ldots \times\left|\mathcal{U}_{M}^{S}\right|$, which can be quite large.

As for the $N$ - and $(N, R)$-heuristic policies, the static policies specify only a pricing policy. Hence, for implementation, we assume the bandwidth-based drop-off policy is used.

\section{Experiments}

In this section, we present the results of a numerical study, which we conducted in order to: (1) obtain additional insights, beyond the analytical results provided above, on the behavior of the optimal prices (Section 6.2); (2) test the performance of the static myopic pricing policy relative to the optimal policy (Section 6.3); (3) test the performance of the proposed dynamic pricing heuristics relative to the optimal dynamic pricing policy (Section 6.4 ) and the optimal static pricing policy (Section 6.5); and (4) evaluate the value of using the optimal drop-off policy compared to the bandwidth-based drop-off policy (Section 6.6).

The analytical results presented in this paper were derived in the context of a discounted MDP. However, because our MDP is unichain, all of the results also hold for the analogous long-run average-value problems. Unichain implies that, given the use of any stationary policy, there exists a state $(\mathbf{N}, \mathbf{R})=(0, \ldots, 0)$ to which there is a positive probability of returning within a finite number of transitions starting from any initial state. When $\gamma=0$ in our MDP formulations, the VIA can be used to determine the optimal long-run average 
value (see Section 8.5 in Puterman (1994)). In the literature, the average-value criterion is generally preferred when conducting numerical experiments because average value does not depend on the initial state, and thus the numerical results are easier to interpret. Therefore, in this section, we present numerical results using the average-value criterion.

To evaluate the value function under the heuristics, we relied on simulation. While the proposed heuristics can be used to find the dynamic pricing policy for the aggregate state space (which we use as our heuristic policy), the resulting value function does not represent the long-run average profit from implementing the heuristic in the complete state-space setting. Thus, we simulate the implementation of the heuristic dynamic pricing policies in the full state-space setting to evaluate the value function. We ran each simulation for a run length of 30 years with 40 replications. We report the simulated average value, which is the average of the long-run average value estimates across the 40 replications.

\subsection{Experiment Configurations}

We first describe the three sets of experiments we conducted. In the first, we set the number of the channel and customer classes such that the state space of the MDP is relatively small and thus VIA can successfully find the optimal policy. We use this experiment to study the behavior of the optimal prices and to compare the performance of the optimal policy and the heuristic policies. In the second, we set the channel and customer classes to larger values, which results in a larger state space, making it impossible to solve the VIA to find the optimal policy. We use this experiment to compare the performance of the proposed dynamic pricing heuristic policy to that of the (static) myopic policy and to determine when the proposed heuristic offers the most value. In the third, we consider a realisticallysized problem in which there are 32 distinct customer classes. We use this experiment to further investigate the performance of the proposed dynamic pricing heuristic.

In all three sets of experiments, the price set is $\mathcal{U}=\{1.0,1.1, \ldots, 5.9,6.0\}$. To model the price sensitivities, we use the same approach as GS, i.e., we calculate the price acceptance probabilities as $p_{i \ell}\left(w_{i \ell}\right)=\left(\frac{w_{i, \max }-w_{i \ell}}{w_{i, \max }-w_{i, \min }}\right)^{\beta_{i}}$, where $w_{i, \max }=\max _{\ell} w_{i \ell}$ and $w_{i, \min }=\min _{\ell} w_{i \ell}$. Here, larger $\beta_{i}$ implies that customer class $i$ is more price sensitive.

6.1.1. Experiment 1 This set of experiments uses configurations with $D=2$ channel classes and $M=2$ customer classes. We define three cases for the channel classes, which we label as I, II and III. As shown in Table 1, the expected availability times of the channels, 
Batur et al.: Dynamic Pricing of Wireless Internet

Article submitted to Manufacturing \& Service Operations Management; manuscript no. (Please, provide the manuscript number!) 25

$1 / \omega_{1}$ and $1 / \omega_{2}$, are identical for all three cases. However, the expected unavailability time in channel class $1,1 / \phi_{1}$, increases from case I to case III, while the opposite holds for $1 / \phi_{2}$. Also, in all three cases, $R_{\max , 2}$ is larger than $R_{\max , 1}$. As a result, the expected capacity increases from case I to III. This allows us to understand how the performance of the optimal and heuristic policies changes as the expected capacity increases.

Table 1 Channel characteristics in Experiment 1

\begin{tabular}{c|c|c|c|c|c|c|} 
& \multicolumn{2}{|c|}{ Channel class 1} & \multicolumn{3}{|c|}{ Channel class 2} \\
Cases & $R_{\text {max }, 1}$ & $1 / \phi_{1}$ & $1 / \omega_{1}$ & $R_{\max , 2}$ & $1 / \phi_{2}$ & $1 / \omega_{2}$ \\
\hline I & 1 & 1 & 3 & 5 & 8 & 3 \\
\hline II & 1 & 4 & 3 & 5 & 4 & 3 \\
\hline III & 1 & 8 & 3 & 5 & 1 & 3 \\
\hline
\end{tabular}

To enable sensitivity analysis, we defined a set of sensitivity parameters, denoted by $\Delta_{j}$, for $j=1, \ldots, 5$. In Experiment 1, we considered the $\Delta_{j}$ values given in the first column of Table 2. The parameters for customer class 1 were fixed to the configuration presented in the second column of the table. The parameters for customer class 2 were varied according to the formulas given in the third column of the table. Thus, we created a set of configurations by changing the properties of customer class 2 , while the properties of customer class 1 remain fixed. The configuration in which the $\left(\Delta_{1}, \Delta_{2}, \Delta_{3}, \Delta_{4}, \Delta_{5}\right)=(0.5,0.5,1.5,1,0.5)$ is referred to as the customer base-case configuration.

Table 2 Customer characteristics in Experiment 1

\begin{tabular}{l|l|l} 
Sensitivity parameters & Customer class 1 & Customer class 2 \\
\hline$\Delta_{1} \in\{0.25,0.5,1\}$ & $r_{1}=0.5$ & $r_{2}=\Delta_{1} r_{1} \in\{0.125,0.25,0.5\}$ \\
\hline$\Delta_{2} \in\{0.1,0.25,0.5\}$ & $\lambda_{1}=10$ & $\lambda_{2}=\Delta_{2} \lambda_{1} \in\{1,2.5,5\}$ \\
\hline$\Delta_{3} \in\{0.5,1,1.5\}$ & $\mu_{1}=5$ & $\mu_{2}=\Delta_{3} \mu_{1} \in\{2.5,5,7.5\}$ \\
\hline$\Delta_{4} \in\{1,2,10\}$ & $K=\max \left\{2^{\text {nd }}\right.$ largest $w_{1 j}, 2^{\text {nd }}$ largest $\left.w_{2 j}\right\}+\Delta_{4}$ \\
\hline$\Delta_{5} \in\{0.1,0.5,1\}$ & $\beta_{1}=3$ & $\beta_{2}=\Delta_{5} \beta_{1} \in\{0.3,1.5,3\}$ \\
\hline
\end{tabular}

A full-factorial design based on the parameter values presented in Tables 1 and 2 gives 729 distinct configurations for Experiment 1. Because the state spaces of these configurations are reasonably small, the VIA can be used to find the optimal policy. 
Batur et al.: Dynamic Pricing of Wireless Internet
26Article submitted to Manufacturing \& Service Operations Management; manuscript no. (Please, provide the manuscript number!)

6.1.2. Experiment 2 These experiments use configurations with $D=3$ channel classes and $M=5$ customer classes. The channel class characteristics are shown in Table 3 .

Table 3 Channel characteristics in Experiments 2 and 3

\begin{tabular}{c|c|c|c} 
Parameters & Channel class 1 & Channel class 2 & Channel class 3 \\
\hline$R_{\max }$ & 1 & 5 & 4 \\
\hline $1 / \omega$ & 3 & 8 & 4 \\
\hline $1 / \phi$ & 8 & 3 & 4 \\
\hline
\end{tabular}

The parameters for the five customer classes are shown in Table 4 . We do not vary $\mathbf{r}$ in the experiments. However, the arrival rates, $\boldsymbol{\lambda}$, service rates, $\boldsymbol{\mu}$, and price sensitivities, $\boldsymbol{\beta}$, are assigned in either ascending or descending order, as specified in Table 4. For example, in the ascending setting for $\boldsymbol{\lambda}$, class 1 is assigned an arrival rate equal to 16, while class 5 is assigned an arrival rate equal to 20 . In contrast, in the descending setting for $\boldsymbol{\lambda}$, class 1 is assigned an arrival rate equal to 20, while class 5 is assigned an arrival rate equal to 16. The overall service rates are also controlled by $\Delta_{3} \in\{4,2,1,0.5\}$, where smaller values of $\Delta_{3}$ represent a smaller ratio of capacity to demand rate. The drop-off fee is generated in a similar way as for Experiment 1, except that there are five customer classes, $i=1, \ldots, 5$, and we fix $\Delta_{4}=1$. We consider a full factorial design for the customer class characteristics. Thus, in total, Experiment 2 consists of a set of $32(=2 \times 4 \times 2 \times 2)$ configurations.

\begin{tabular}{c|c|c} 
Table 4 & \multicolumn{3}{c}{ Customer characteristics in Experiment 2 } \\
Parameters & Ascending & Descending \\
\hline $\mathbf{r}$ & \multicolumn{3}{|c}{$[0.9,0.7,0.5,0.3,0.1]$} \\
\hline $\boldsymbol{\lambda}$ & {$[16,17,18,19,20]$} & {$[20,19,18,17,16]$} \\
\hline $\boldsymbol{\mu}$ & $\Delta_{3} \times[1,2,3,4,5]$ & $\Delta_{3} \times[5,4,3,2,1]$ \\
\hline $\boldsymbol{\beta}$ & {$[1,1.5,2,2.5,3]$} & {$[3,2.5,2,1.5,1]$} \\
\hline
\end{tabular}

6.1.3. Experiment 3 In practice, it is likely that the number of customer classes will be significantly larger than the five classes considered in Experiment 2. Therefore, we constructed a single configuration with a more realistic number of distinct customer classes. Customer classes are defined according to the customers' bandwidth requirement, arrival 


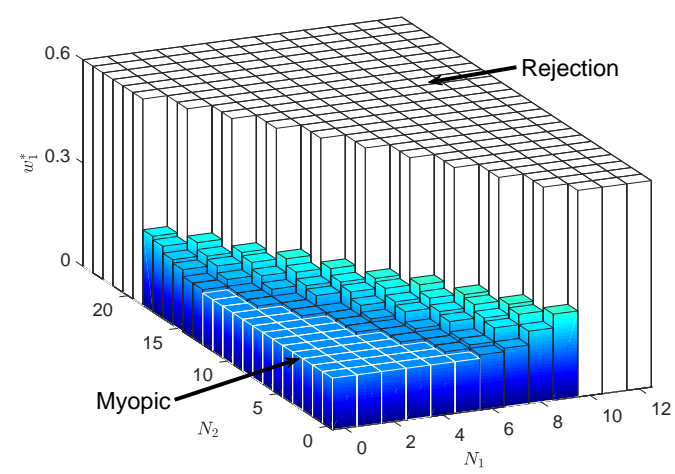

(a) $w_{1}^{*}$ by $N_{1}$ and $N_{2}\left(R_{1}=1, R_{2}=4\right)$

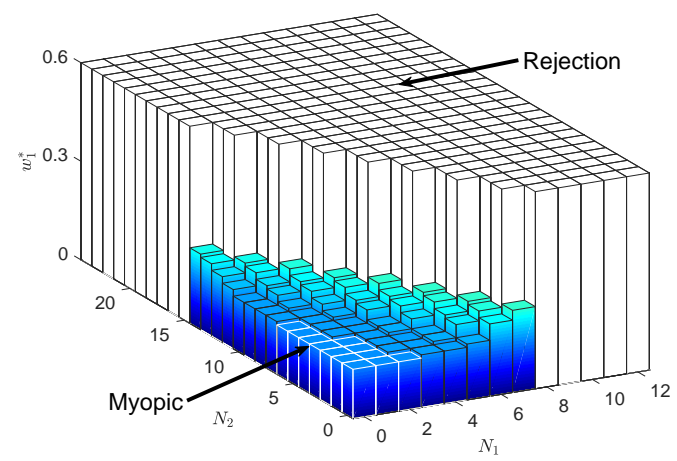

(c) $w_{1}^{*}$ by $N_{1}$ and $N_{2}\left(R_{1}=0, R_{2}=4\right)$

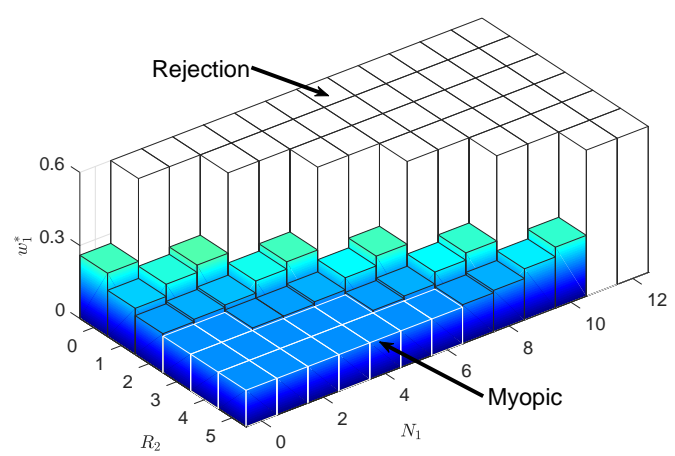

(b) $w_{1}^{*}$ by $N_{1}$ and $R_{2}\left(N_{2}=2, R_{1}=1\right)$

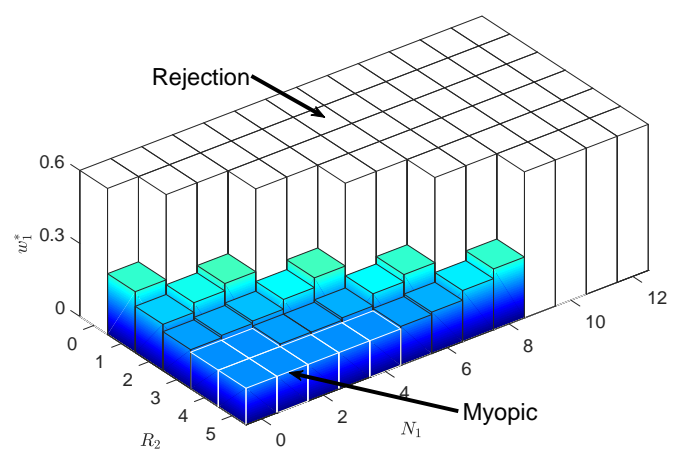

(d) $w_{1}^{*}$ by $N_{1}$ and $R_{2}\left(N_{2}=2, R_{1}=0\right)$

Figure 1 Optimal discounted prices $w_{1}^{*}$ (customer base-case and channel case I configuration in Experiment 1)

rate, service rate, and price sensitivity. We considered $r_{i} \in\{0.0625,0.125,0.25,0.5\}, \lambda_{i} \in$ $\{10,50\}, \mu_{i} \in\{12.5,37.5\}$, and $\beta_{i} \in\{0.9,9\}$. Our experiment used the $M=32(=4 \times 2 \times$ $2 \times 2$ ) customer classes that result from considering all possible combinations of these parameter values. In this experiment, we used the $D=3$ channel classes given in Table 3 .

\subsection{Properties of the Optimal Dynamic Pricing Policies}

In Figure 1, we show how the optimal discounted price for customer class $1, w_{1}^{*}$, changes as the state changes, for the customer base-case configuration and channel case I configuration in Experiment 1. For example, in Figure 1(a), we see how $w_{1}^{*}$ changes as $N_{1}$ and $N_{2}$ change when $R_{1}=1$ and $R_{2}=4$. When $N_{1}$ and $N_{2}$ are large relative to $R_{1}$ and $R_{2}$, the reject price is posted. This is because either $\mathrm{i}$ ) the remaining capacity is not sufficient to admit the arriving class 1 customer; or ii) the remaining capacity is sufficient, but the optimal policy 
28Article submitted to Manufacturing \& Service Operations Management; manuscript al.: Dynamic Pricing of Wireless Internet reserves that remaining capacity for a potentially more profitable future customer. The lowest price posted is the myopic price, consistent with Corollary 1 .

Although Figures 1(a) and 1(c) suggest that the optimal prices are nondecreasing in $N_{1}$ and $N_{2}$, the results of other configurations indicate that this does not always hold:

OBSERVATION 1. The optimal discounted prices are usually, but not always, nondecreasing in the number of customers that have been admitted to the system.

If we look at all 729 configurations that we tested, we find 486 configurations for which $w_{i}^{*}$, in some states, decreases when $N_{i}$ increases. These violations occur for configurations in which $r_{1}>r_{2}$, and they always occur for customer class 2 . When we look at the specific violations, we observe that $w_{2}^{*}$ in states where, for example, $N_{2}=0$ is sometimes larger than that in a state with $N_{2}=1$. We believe this is mainly due to the difference in the bandwidth requirements of the two customer classes, although the different service rates may also play a role, as explained in Theorem 3. For example, suppose $r_{1}=2 r_{2}$. In this case, when $N_{2}$ is an even number, such as $N_{2}=0$, accepting a customer from class 2 may in the future take the model to a state in which the remaining capacity is enough only to admit a class 2 customer, but not enough to admit a class 1 customer. A similar issue does not apply when $N_{2}$ is odd, such as $N_{2}=1$. Örmeci and Burnetas (2004) find similar violations of monotonicity for loss systems in which jobs arrive in batches of random size when partial batch acceptance is not allowed and the number of servers is fixed. Specifically, they find examples in which the system may reject arriving jobs in order to wait for a job with a batch size that will just make use of all of the available resources (servers).

Corollary 2 indicates that the optimal discounted prices are nonincreasing in $R_{d}$, i.e., $w_{i}^{*}(N, \mathbf{R}) \geq w_{i}^{*}\left(N, \mathbf{R}+\mathbf{e}_{d}^{D}\right)$ for the case of similar customer classes. Our numerical experiments indicate that this result holds in general, as shown in Figures $1(\mathrm{~b})$ and 1(d).

OBSERVATION 2. The optimal discounted prices are nonincreasing in the number of available channels.

Finally, the figures in Appendix $\mathrm{G}$ show how the optimal average values change as each sensitivity parameter, $\Delta_{j}$, changes in Experiment 1 . The results are consistent with the structural results proven in Section 4 . 
Batur et al.: Dynamic Pricing of Wireless Internet

Article submitted to Manufacturing $\&$ Service Operations Management; manuscript no. (Please, provide the manuscript number!) 29

\subsection{Performance of the Myopic Pricing Policy}

We next consider the performance of the myopic policy relative to the optimal policy. Figure 2 presents the simulated average values of the optimal and myopic policies for the customer base-case configuration in Experiment 1 as the arrival rate of customer class $1, \lambda_{1}$, decreases and the channel-class case changes. As expected, the average value of the optimal policy is always larger than the average value of the myopic policy. In addition, Observation 3 provides insights that are critical for the dynamic spectrum access application setting, in which scarcity of capacity (bandwidth) is likely to be a significant issue.

OBSERVATION 3. The myopic policy offers performance similar to that of the optimal policy when the customer arrival rate is small and capacity is high (recall that the expected capacity increases from case I to III). Performance degradation from the myopic policy is largest when the ratio of capacity to demand rate is low.

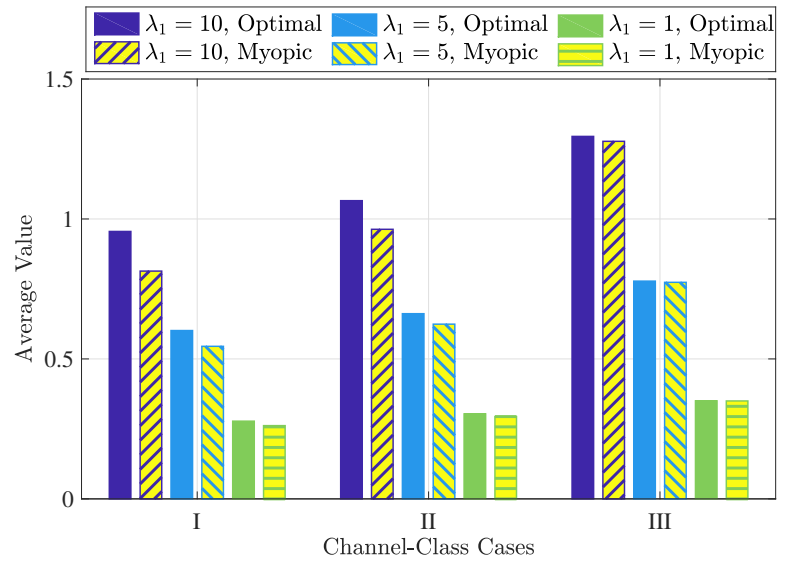

Figure 2 Simulated average values across 40 replications (customer base-case configuration in Experiment 1)

Figure 2 shows the customer base-case configuration. When all configurations are considered, the percentage value loss of the myopic policy with respect to the optimal policy ranges from $1.1 \%$ to $385.3 \%$, with an average of $36.2 \%$.

In order to explain the performance gap between the optimal and myopic policies, we analyzed the customer rejection ratios for the optimal and myopic policies, as shown in Figure 3. The customer rejection ratio is the percentage of customers that reject the posted price when there is sufficient capacity in the system. We have the following observation:

OBSERVATION 4. The customer rejection ratios for the myopic policy are always smaller than those for the optimal policy. The rejection ratios for the myopic policy decrease and 
30 Batur et al.: Dynamic Pricing of Wireless Internet 30Article submitted to Manufacturing \& Service Operations Management; manuscript no. (Please, provide the manuscript number!)

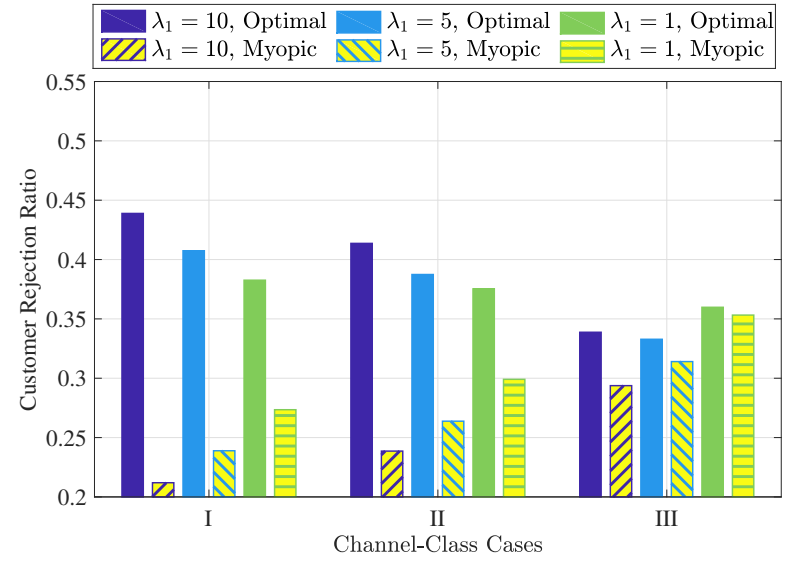

(a) Customer class 1

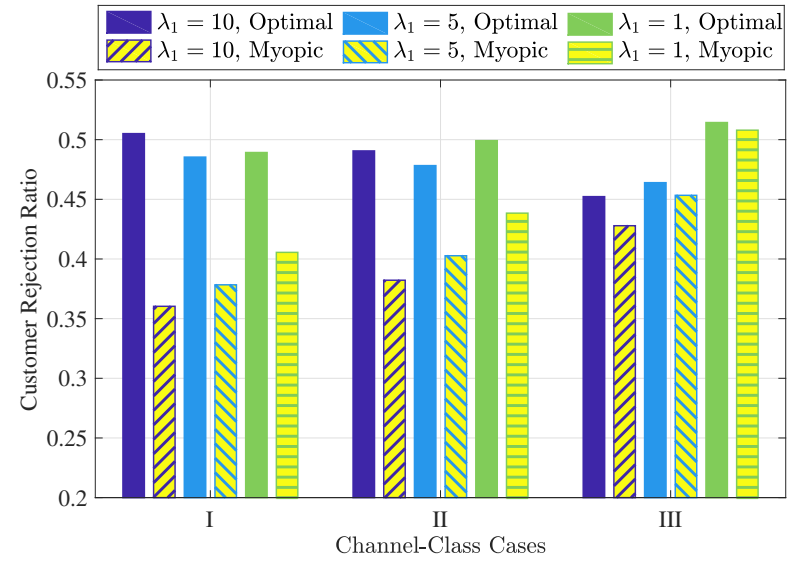

(b) Customer class 2

Figure 3 Rejection ratios averaged across 40 replications (customer base-case configuration in Experiment 1)

deviate more from those of the optimal policy as the customer arrival rate increases and as capacity decreases (recall that the expected capacity increases from case I to III).

The intuition behind these results is that the myopic policy always posts the myopic price if capacity exists to admit the arriving customer. This leads to lower rejection ratios, which ultimately leads to a high rate of customer drop-off, as well as a high rate of nocapacity rejection, i.e., rejections that occur when there is no capacity available in the system to accept the arriving customer. In contrast, the optimal policy posts prices that are higher than the myopic prices when doing so is necessary to prevent congestion and future customer drop-offs. This leads to higher rejection ratios, but also higher profits.

\subsection{Performance of the Proposed Dynamic Pricing Heuristics}

We next compare the performance of the three dynamic pricing heuristics, the $N_{-}, R-$, and $(N, R)$-heuristics for Experiment 1. The average value estimated from the simulation of the heuristic policies is compared with the optimal average value, $\bar{v}$, obtained from the VIA. We report two kinds of percentage value losses. When the optimal policy is simulated, the percentage value loss, $X$, is defined as $X=\left(\bar{v}-\bar{V}_{\text {opt }}\right) / \bar{v}$, where $\bar{V}_{\text {opt }}$ is the average value obtained from simulation of the optimal policy. This percentage value loss shows the accuracy of the simulation model. In contrast, when one of the heuristic policies is simulated, the percentage value loss, $X$, is defined as $X=\left(\bar{v}-\bar{V}_{\text {heu }}\right) / \bar{v}$, where $\bar{V}_{\text {heu }}$ is the average value obtained from simulation of the heuristic policy. This percentage value loss shows the performance of the evaluated heuristic compared to optimal policy. We compute 
the percentage value loss for each of the 729 configurations and use these 729 observed values to draw an empirical cumulative distribution function $(\mathrm{CDF})$, i.e., $\operatorname{Pr}(X \leq x)$.

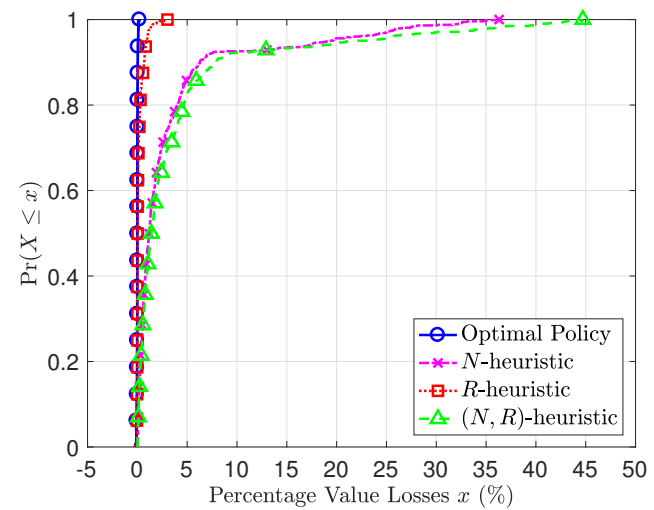

Figure 4 Empirical CDF of percentage value loss in Experiment 1 (across 40 replications)

The empirical CDFs for the optimal policy and the heuristics are shown in Figure 4. For the optimal policy, the percentage error induced by random simulations is close to zero, which validates the simulation model. The CDFs of the heuristics are always to the right of that for the optimal policy. The differences between the CDFs of the heuristics and the optimal policy show the value loss due to aggregating customer and/or channel classes.

OBservation 5. The performance of the R-heuristic is close to that of the optimal policy, i.e., aggregation of channel classes results in only a slight performance degradation. The $N$ - and $(N, R)$-heuristics result in more significant performance degradation. While they provide similar performance in most configurations, in $10 \%$ of the configurations the $(N, R)$-heuristic results in larger loss than the $N$-heuristic due to the fact that it aggregates channel classes, while the $N$-heuristic does not. Overall, the results indicate that aggregating customer classes results in a greater loss in value than aggregating channel classes.

In Appendix $\mathrm{H}$, the CDFs for each of the heuristics are analyzed for six key problem parameters in order to demonstrate which problem parameters have the greatest impact on the performance of the heuristics. The following observation summarizes the results:

OBSERVATION 6. The performance of the $R$-heuristic is robust to the changes in the key parameters. The performance of the $N$ - and $(N, R)$ - heuristics is not affected by the service rate, arrival rate, and price sensitivity. However, the heuristic performance degrades when the bandwidth requirement for class 2 customers is small (which implies that there is a 
Batur et al.: Dynamic Pricing of Wireless Internet 32Article submitted to Manufacturing \& Service Operations Management; manuscript no. (Please, provide the manuscript number!)

significant difference in bandwidth requirements for the classes), when the drop-off fee is large, and in channel-class case I (which represents low expected capacity).

While Observation 5 states that the $R$-heuristic out-performs the $N$ - and $(N, R)$ heuristics, it is also important to consider computational complexity. In practice, it is likely that there will be many customer classes, which will lead to a high-dimension state space. In that case, channel-class aggregation may not be sufficient to enable implementation of VIA, i.e., customer-class aggregation may also be needed to reduce the size of the state space to a feasible level. Thus, in the remainder of the paper, we focus on the performance of the $(N, R)$-heuristic, which combines both customer- and channel-class aggregation.

Table 5 Percentage value gain of $(N, R)$-heuristic over myopic policy in Experiment 2 (across 40 replications)

\begin{tabular}{c|c|c|c|c|c|c|c}
\multicolumn{2}{c|}{$\Delta_{3}=4$} & \multicolumn{2}{c|}{$\Delta_{3}=2$} & \multicolumn{2}{c|}{$\Delta_{3}=1$} & \multicolumn{2}{c}{$\Delta_{3}=0.5$} \\
\hline Avg. & Std. Err. & Avg. & Std. Err. & Avg. & Std. Err. & Avg. & Std. Err. \\
\hline $0.3 \%$ & $0.7 \%$ & $5.2 \%$ & $2.2 \%$ & $31.3 \%$ & $5.5 \%$ & $998.7 \%$ & $723.3 \%$ \\
\hline
\end{tabular}

We next evaluate the performance of the $(N, R)$-heuristic for Experiment 2, which has a larger state space. For these configurations, it is computationally infeasible to solve VIA to find the optimal policy. Therefore, to evaluate the performance of the $(N, R)$-heuristic in Experiment 2, we compare it to the non-state-dependent myopic policy. We report the percentage value gain of the heuristic policy over the myopic policy, which is defined as $\left(\bar{V}_{\text {heu }}-\bar{V}_{\text {myo }}\right) / \bar{V}_{\text {myo }}$, where $\bar{V}_{\text {heu }}$ and $\bar{V}_{\text {myo }}$ are the simulated average values of the $(N, R)-$ heuristic and myopic policies, respectively. Table 5 shows the average of this percentage value gain for each of the four levels of capacity $\left(\Delta_{3}\right)$ in Experiment 2 . The table also reports the corresponding standard errors. Our main results are as follows:

OBSERVATION 7. Using the $(N, R)$-heuristic pricing policy adds the most value relative to the myopic policy when capacity is scarce $\left(\Delta_{3}\right.$ small), while the value of using the $(N, R)$ heuristic is minimal when capacity is abundant $\left(\Delta_{3}\right.$ large).

The dynamic $(N, R)$-heuristic and the static myopic policy differ significantly in terms of how they manage capacity. This can be seen by considering the customer rejection ratio, no-capacity rejection ratio, and drop-off ratio in Experiment 2, as shown in Figure 5. The figure shows results for customer classes 1 and 5; a similar pattern holds for the other classes. Figures 5(b), 5(c), 5(e), and 5(f) use a logarithmic scale to clearly show the results. 


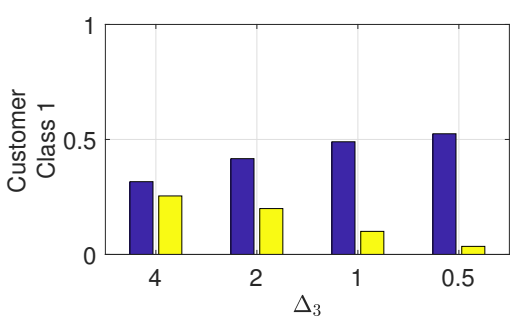

(a) Customer rejection ratio

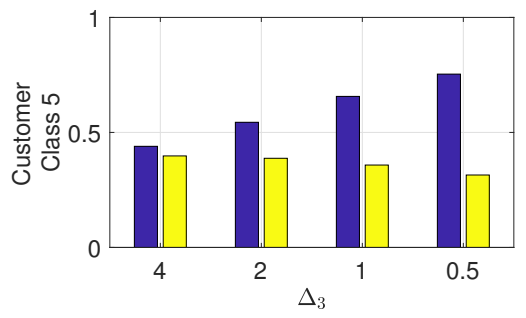

(d) Customer rejection ratio

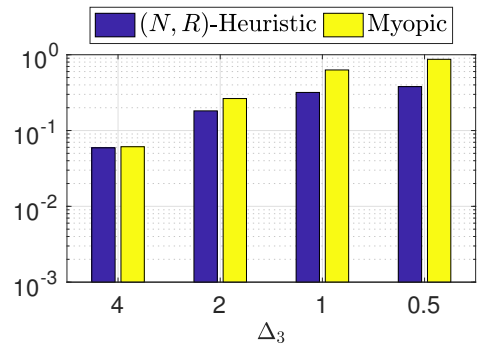

(b) No-capacity rejection ratio

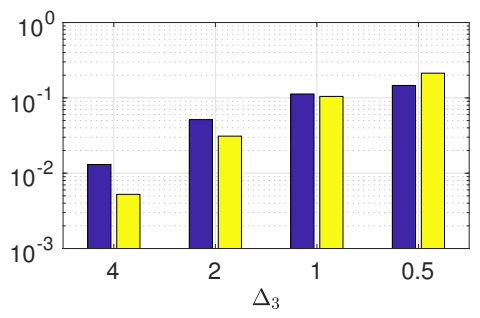

(e) No-capacity rejection ratio

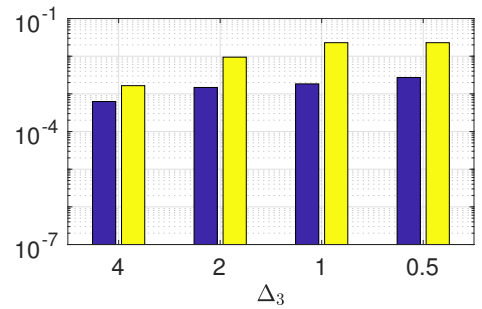

(c) Drop-off ratio

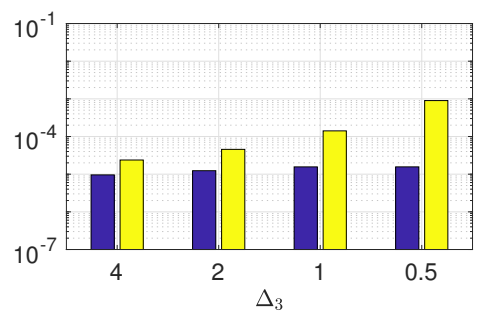

(f) Drop-off ratio

Figure 5 Customer rejection ratio, no-capacity rejection ratio, and drop-off ratio averaged across 40 replications in Experiment 2 (The configuration of all parameters are in descending order in Table 4).

ObSERVATION 8. As the ratio of capacity to demand rate decreases (as $\Delta_{3}$ decreases):

- Under the myopic policy, the reject prices are offered more often due to a lack of capacity. This leads to an increase in no-capacity rejection ratios, a decrease in customer rejection ratios, and an increase in the drop-off ratios.

- Under the $(N, R)$-heuristic, higher prices are posted, leading to the higher customer rejection ratios. Since more customers are rejecting these higher prices at admission, the no-capacity rejection ratio and the drop-off ratio are less than those for the myopic policy. Overall, a higher value is achieved.

Observations 7 and 8 demonstrate the importance of dynamically managing pricing and admission, by using the dynamic (state-dependent) $(N, R)$-heuristic policy rather than the static myopic policy, particularly in settings with scarce capacity.

Finally, we also compared the performance of the $(N, R)$ - and myopic heuristics for Experiment 3, which has a realistically-sized state space. We found that the simulated average value of the $(N, R)$-heuristic was $22.3 \%$ higher than that of myopic policy.

\subsection{Performance of the Optimal Static Pricing Policy}

Finally, we evaluate the performance of the optimal static pricing policy. As described in Section 5.4, finding the optimal static prices is computationally challenging. Therefore, we 
Batur et al.: Dynamic Pricing of Wireless Internet
34Article submitted to Manufacturing \& Service Operations Management; manuscript no. (Please, provide the manuscript number!) determined the optimal static pricing policy for 27 of the 729 experimental configurations defined in Experiment 1. For these problems, we found that the percentage value loss for each heuristic, relative to the optimal average value, was as follows: $1.69 \%$ for $N$-heuristic, $0.51 \%$ for the $R$-heuristic, $2.42 \%$ for the $(N, R)$-heuristic, $30.84 \%$ for the myopic policy, and $10.23 \%$ for the optimal static pricing policy. In all 27 configurations, all of the dynamic pricing heuristics outperformed the optimal static pricing policy. From these experiments, we have the following observation:

OBSERVATION 9. When capacity is scarce, using dynamic pricing to manage admission of customers becomes essential.

Dynamic pricing adds value relative to static pricing when customers are highly price sensitive because dynamic pricing enables the SP to offer lower prices when the remaining capacity is sufficiently large. Overall, we conclude that, in practical settings for which capacity is expected to be tight, the dynamic pricing heuristics provide both better performance and reduced computational time compared to the optimal static pricing policy.

\subsection{Comparison of Optimal and Bandwidth-Based Drop-Off Functions}

As discussed in Section 4, the optimal drop-off policy is not identical to the bandwidthbased drop-off policy. However, the latter is easier to compute and to implement than the former. In addition, the proposed $(N, R)$-heuristic uses the bandwidth-based drop-off policy for implementation. Therefore, we used Experiment 1 to study how much the SP could lose from implementing the bandwidth-based drop-off policy, rather than the optimal drop-off policy. To do so, we compared the average value when the MDP in Section 3.2 is solved using the optimal drop-off policy compared to when the MDP is solved using the bandwidth-based drop-off policy. The results are summarized in the following observation:

OBSERVATION 10. The percentage value loss from using the bandwidth-based drop-off policy, rather than the optimal drop-off policy, is less than $0.4 \%$ for each of the 729 configurations in Experiment 1.

\section{Conclusion}

Inspired by new developments in dynamic spectrum access solutions for improved management of wireless networks, we study the optimal dynamic pricing policy for a service 
Batur et al.: Dynamic Pricing of Wireless Internet

Article submitted to Manufacturing \& Service Operations Management; manuscript no. (Please, provide the manuscript number!) 35

provider who operates in a setting in which both demand (customers wanting to access the Internet) and capacity (available bandwidth) are stochastic. We characterize the behavior of the optimal pricing policy as a function of the system state (number of customers in the system and number of available channels) and of the input parameters. Further, for the setting in which both customer arrivals and capacity are stochastic, we have demonstrated the value of using a dynamic, i.e., state-dependent, pricing policy compared to static, i.e., non-state-dependent, policies. The previous literature, i.e., GS, has studied systems with stochastic customer arrivals and fixed capacity. This previous work has focused on analytically and numerically characterizing the conditions under which static myopic policies perform optimally or close-to-optimally. Like this previous work, we find that using the optimal dynamic pricing policy, rather than the myopic policy, becomes more critical when capacity is scarce. In addition, when capacity is scarce, the optimal pricing policy outperforms the myopic policy in terms of the no-capacity rejection ratios and customer drop-off ratios, which can be viewed as measures of quality-of-service and access.

Compared to the setting studied in GS, our problem setting is more dynamic, i.e., our setting has dynamic capacity, as channels become available and unavailable for use. Therefore, identifying good state-dependent heuristic pricing policies, i.e., policies that will adjust the price based on the actual capacity, as well as the number of customers currently being served, will be of greater value for our problem setting than for the GS setting with constant capacity. Thus, we develop and propose the $(N, R)$-heuristic, in which the pricing policy is dependent on the aggregate capacity, as well as the aggregate number of customers being served. While still state-dependent, this policy is computationally much more tractable, and easier to implement in practical settings, than both the optimal dynamic pricing policy and the optimal static pricing policy. We find that the $(N, R)$-heuristic can provide a significant performance improvement relative to the myopic and optimal static pricing policies, particularly when capacity is scarce. In the dynamic spectrum access application, scarcity of capacity (bandwidth) is likely to be a significant issue. Thus, in practice, the dynamic heuristic pricing policy is likely to provide significantly improved profits for the service provider, as well as improved quality-of-service (lower drop-off ratios) and access (lower no-capacity rejection ratios), compared to the static pricing policies.

\section{Acknowledgments}

This material was supported by the National Science Foundation under Grant Number CNS-1247941. 
Batur et al.: Dynamic Pricing of Wireless Internet
36Article submitted to Manufacturing \& Service Operations Management; manuscript no. (Please, provide the manuscript number!)

\section{References}

Aktaran-Kalayci, T., H. Ayhan. 2009. Sensitivity of optimal prices to system parameters in a steady-state service facility. European Journal of Operational Research, 193, 120-128.

Altman, E., T. Jimenez, G. Koole. 2001. On optimal call admission control. IEEE Trans. Comm., 49 (9) 1659-1668.

Beltran, F., S. K. Ray, J. A. Gutierrez. 2016. Understanding the current operation and future roles of wireless networks: Co-existence, competition and co-operation in the unlicensed spectrum bands. IEEE Journal on Selected Areas in Communications, 34(11) 2829-2837.

Bhattarai, S., J.-M. Park, B. Gao, K. Bian, W. Lehr. 2016. An Overview of Dynamic Spectrum Sharing: Ongoing Initiatives, Challenges, and a Roadmap for Future Research. IEEE Transactions on Cognitive Communications and Networking, 2(2), 110-128.

Beltran, F., S. K. Ray, J. A. Gutierrez. 2016. An overview of pricing models for revenue management. Manufacturing \& Service Operations Management, 5(3), 203-229.

Cachon, G. P., K. M. Daniels, R. Lobel. 2017. The role of surge pricing on a service platform with selfscheduling capacity. To be published in Manufacturing \& Service Operations Management. Retrieved from https://doi.org/10.1287/msom.2017.0618.

Carrizosa, E., E. Conde, M. Munoz-Marquez. 1998. Admission policies in loss queueing models with heterogeneous arrivals. Management Science, 44, 311-320.

Chang, C.-H., P. Lin, J. Zhang, J.-Y. Jeng. 2015. Time dependent adaptive pricing for mobile Internet access. The Fourth IEEE Workshop on Smart Data Pricing, 540-545.

Chen, B. X. 2012. Carriers Warn of Crisis in Mobile Spectrum. Retrieved from http://www.nytimes.com/ 2012/04/18/technology/mobile-carriers-warn-of-spectrum-crisis-others-see-hyperbole. html.

Cil, E. B., E. L. Örmeci, F. Karaesmen. 2009. Effects of system parameters on the optimal policy structure in a class of queueing control problems. Queueing Systems, 61, 273-304.

Cisco Visual Networking Index: Global Mobile Data Traffic Forecast Update, 2015-2020 White Paper. 2016. Retrieved from http://www.cisco.com/c/en/us/solutions/collateral/service-provider/ visual-networking-index-vni/mobile-white-paper-c11-520862.html.

Federal Communications Commission. 2002. FCC Spectrum Policy Task Force: Report of the Spectrum Efficiency Working Group. Retrieved from https://transition.fcc.gov/sptf/files/ SEWGFinalReport_1.pdf

Federal Communications Commission. 2010. Second Memorandum Opinion and Order. Retrieved from http: //hraunfoss.fcc.gov/edocs_public/attachmatch/FCC-10-174A1.pdf.

Federal Communications Commission. 2015. Amendment of the Commissions Rules with Regard to Commercial Operations in the 3550-3650 MHz Band. Retrieved from https://apps.fcc.gov/edocs_public/ attachmatch/FCC-15-47A1.pdf.

Gans, N., S. Savin. 2007. Pricing and capacity rationing for rentals with uncertain durations. Management Science, 53 (3), 390-407. 
Batur et al.: Dynamic Pricing of Wireless Internet

Article submitted to Manufacturing $\mathcal{E}$ Service Operations Management; manuscript no. (Please, provide the manuscript number!)37

Gayon, J.-P., I. Talay-Deḡirmenci, F. Karaesmen, L. Örmeci. 2009. Optimal pricing and production policies of a make-to-stock system with fluctuating demand. Probability in the Engineering and Informational Sciences, 23, 205-230.

Gurvich, I., M. Lariviere, A. Moreno-Garcia. 2017. Operations in the on-demand economy: Staffing services with self-scheduling capacity. Working paper. Retrieved from http://www.kellogg.northwestern. edu/faculty/lariviere/research/GLM_Revised_WithNames.pdf.

Harrison, K., S. M. Mishra, A. Sahai. 2010. How Much White-Space Capacity Is There? Proc. IEEE DySPAN 2010, 1-10.

Huang, H.-S., S.-C. Hu, P.-H. Lee, Y.-C. Tseng. 2016. An adaptive Paris Metro Pricing scheme for mobile data networks. International Journal of Network Management, 26, 422-434.

Ibrahim, R. 2017. Managing queueing systems where capacity is random and customers are impatient. Working paper. Retrieved from http://www.roubaibrahim.com/SelfSchedule.pdf.

LTE-U Forum. 2015. LTE-U Technical Report, Coexistence Study for LTE-U SDL. Retrieved from http://www.lteuforum.org/uploads/3/5/6/8/3568127/lte-u_forum_lte-u_technical_report_ v1.0.pdf

Lippman, S. 1975. Applying a new device in the optimization of exponential queueing systems. Operations Research, 23 (4), 687-710.

Miller, B. L. 1969. A queueing reward system with several customer classes. Management Science, 16 (3), 234-245.

Mutlu, H., M. Alanyali, D. Starobinski. 2009. Spot pricing of secondary spectrum access in wireless cellular networks. IEEE/ACM Transactions on Networking, 17 (6), 1794-1804.

Örmeci, E. L., A. Burnetas. 2004. Admission control with batch arrivals. Operations Research Letters, 32, 448-454.

Paschalidis, I. Ch., J. N. Tsitsiklis. 2000. Congestion-dependent pricing of network services. IEEE/ACM Transactions on Networking, 8 (2), 171-184.

Puterman, M. L. 1994. Markov Decision Processes, Wiley, New York.

Righter, R. 1994. Scheduling in Stochastic Orders and Their Applications. Editors M. Shaked and J. G. Shanthikumar. Academic Press.

Ross, K. W., D. H. K Tsang. 1989. The stochastic knapsack problem. IEEE Transactions on Communications, 37 (7), 740-747.

Tsai, Y.-C., Y.-D. Cheng, C.-W. Wu, Y.-T. Lai, W.-H. Hu, J.-Y. Jeng, Y.-C. Tseng. 2017. Time-dependent smart data pricing based on machine learning. Canadian Conference on Artificial Intelligence, 103-108.

Turhan, A., M. Alanyali, D. Starobinski. 2012. Optimal Admission Control of Secondary Users in Preemptive cognitive radio networks. Proceedings of the 10th InteNrational Symposium on Modeling and Optimization in Mobile, Ad Hoc, and Wireless Networks (WiOpt), 138-144.

Ulukus, M. Y., R. Güllü, L. Örmeci. 2011. Admission and termination control of a two-class loss system. Stochastic Models, 27, 2-25.

Whitt, W. 2011. Staffing a call center with with uncertain arrival rate and absenteeism. Production and Operations Management, 15(1), 88-102. 
38 $\quad$ Batur et al.: Dynamic Pricing of Wireless Internet 38Article submitted to Manufacturing \& Service Operations Management; manuscript no. (Please, provide the manuscript number!)

Yahav, I., I. Karaesmen, L. Raschid. 2013. Managing on-demand computing services with heterogeneous customers. Proc. of the 2013 Winter Simulation Conference, 5-16.

Zhang, X., E. Knightly. 2015. WATCH: WiFi in Active TV Channels. Proc. of ACM MobiHoc 2015, 7-16.

Zhang, L., W. Wu, D. Wang. 2014. Time dependent pricing in wireless data networks: flat-rate vs. usagebased schemes. Proc. of IEEE INFOCOM '14, 700-708.

Zhao, Z., S. Weber, J. C. de Oliveira. 2010. Admission control and preemption policy design of multi-class computer networks. Proc. of the 44th Conference on Information Sciences and Systems (CISS), 1-6.

Zhao, Z., M. C. Vuran, D. Batur, and E. Ekici. 2014. Ratings for spectrum: Impacts of TV viewership on TV whitespace. Proc. of IEEE Globecom '14, 941-947. 
Batur et al.: Dynamic Pricing of Wireless Internet
B.0Article submitted to Manufacturing \&s Service Operations Management; manuscript no. (Please, provide the manuscript number!)

\section{A. Proof of Theorem 1}

Let $A_{i 1}$ and $A_{i 2}$ be two opportunity costs related for customer class $i$ such that $A_{i 1}>A_{i 2}$. Define the index of the optimal price by $\ell_{i}^{*}\left(A_{i}\right)=\arg \max _{\ell}\left[p_{i \ell}\left(-A_{i}+w_{i \ell}\right)\right]$. Then

$$
\begin{aligned}
g_{i}^{*}\left(A_{i 1}\right)=p_{i \ell_{i}^{*}\left(A_{i 1}\right)}\left(-A_{i 1}+w_{i \ell_{i}^{*}\left(A_{i 1}\right)}\right) & \leq p_{i \ell_{i}^{*}\left(A_{i 1}\right)}\left(-A_{i 2}+w_{i \ell_{i}^{*}\left(A_{i 1}\right)}\right) \\
& \leq p_{i \ell_{i}^{*}\left(A_{i 2}\right)}\left(-A_{i 2}+w_{i \ell_{i}^{*}\left(A_{i 2}\right)}\right)=g_{i}^{*}\left(A_{i 2}\right) .
\end{aligned}
$$

Hence, $g_{i}^{*}\left(A_{i}\right)$ is nonincreasing in $A_{i}$.

Next, we want to show that $w_{i}^{*}\left(A_{i}\right)$ is nondecreasing in $A_{i}$. Then we need to show that $\ell_{i}^{*}\left(A_{i 1}\right) \geq \ell_{i}^{*}\left(A_{i 2}\right)$. We prove this result by contradiction, i.e., we assume $\ell_{i}^{*}\left(A_{i 1}\right)<\ell_{i}^{*}\left(A_{i 2}\right)$, which implies $p_{i \ell_{i}^{*}\left(A_{i 1}\right)}>p_{i \ell_{i}^{*}\left(A_{i 2}\right)}$. By definition, $\ell_{i}^{*}\left(A_{i 1}\right)$ and $\ell_{i}^{*}\left(A_{i 2}\right)$ satisfy the following:

i. $p_{i \ell_{i}^{*}\left(A_{i 1}\right)}\left(-A_{i 1}+w_{i \ell_{i}^{*}\left(A_{i 1}\right)}\right) \geq p_{i \ell_{i}^{*}\left(A_{i 2}\right)}\left(-A_{i 1}+w_{i \ell_{i}^{*}\left(A_{i 2}\right)}\right)$ and ii. $p_{i \ell_{i}^{*}\left(A_{i 2}\right)}\left(-A_{i 2}+w_{i \ell_{i}^{*}\left(A_{i 2}\right)}\right) \geq$ $p_{i \ell_{i}^{*}\left(A_{i 1}\right)}\left(-A_{i 2}+w_{i \ell_{i}^{*}\left(A_{i 1}\right)}\right)$. These two imply that

$$
A_{i 1}\left(p_{i \ell_{i}^{*}\left(A_{i 1}\right)}-p_{i \ell_{i}^{*}\left(A_{i 2}\right)}\right) \leq p_{i \ell_{i}^{*}\left(A_{i 1}\right)} w_{i \ell_{i}^{*}\left(A_{i 1}\right)}-p_{i \ell_{i}^{*}\left(A_{i 2}\right)} w_{i \ell_{i}^{*}\left(A_{i 2}\right)} \leq A_{i 2}\left(p_{i \ell_{i}^{*}\left(A_{i 1}\right)}-p_{i \ell_{i}^{*}\left(A_{i 2}\right)}\right),
$$

which implies $A_{i 1} \leq A_{i 2}$ due to $p_{i \ell_{i}^{*}\left(A_{i 1}\right)}-p_{i \ell_{i}^{*}\left(A_{i 2}\right)}>0$. Thus, we have a contradiction.

\section{B. Proof of Theorem 2}

Lemma B1. For $\mathbf{c} \in \mathbb{Z}_{+}^{M}, f(\mathbf{N}+\mathbf{c}, \mathbf{R})-f(\mathbf{N}, \mathbf{R})+c K \geq 0$, where $(\mathbf{N}+\mathbf{c})^{\prime} \mathbf{r} \leq R$.

Proof of Lemma B1. We use induction in this proof. When $c=\mathbf{e}_{i}^{M}$, we get

$$
f\left(\mathbf{N}+\mathbf{e}_{i}^{M}, \mathbf{R}\right)-f(\mathbf{N}, \mathbf{R})+K \geq 0 .
$$

In the following, we show that Equation (B.1) holds. In the $H_{i}$ function in Equation 4), let $\ell_{1}=\arg \max _{\ell}\left[p_{i \ell}\left(f\left(\mathbf{N}+\mathbf{e}_{i}^{M}, \mathbf{R}\right)+w_{i \ell}-f(\mathbf{N}, \mathbf{R})\right)+f(\mathbf{N}, \mathbf{R})\right]$. This implies that $f\left(\mathbf{N}+\mathbf{e}_{i}^{M}, \mathbf{R}\right)+w_{i \ell_{1}}-f(\mathbf{N}, \mathbf{R}) \geq 0$. We can replace $w_{i \ell_{1}}$ by $K$ because $w_{i \ell_{1}}<K$ when $\ell_{1}<L$ by Assumption 3. Hence, Equation (B.1) holds.

Now, assume that $f\left(\mathbf{N}+\mathbf{c}-\mathbf{e}_{i}^{M}, \mathbf{R}\right)-f(\mathbf{N}, \mathbf{R})+(c-1) K \geq 0$. holds. Then we get $f(\mathbf{N}+$ $\left.\mathbf{c}-\mathbf{e}_{i}^{M}, \mathbf{R}\right) \geq f(\mathbf{N}, \mathbf{R})-(c-1) K$. From Equation B.1), we get $f(\mathbf{N}+\mathbf{c}, \mathbf{R})-f(\mathbf{N}+\mathbf{c}-$ $\left.\mathbf{e}_{i}^{M}, \mathbf{R}\right)+K \geq 0$. From the previous two inequalities, we get $f(\mathbf{N}+\mathbf{c}, \mathbf{R})-f(\mathbf{N}, \mathbf{R})+c K \geq 0$. This completes the proof by induction.

Proof of Theorem 2.1. From Equation (6), we define the following $H$ function

$$
\begin{aligned}
& H(\mathbf{N}, \mathbf{R})=\sum_{i=1}^{M} \mu_{i} N_{i} f\left(\mathbf{N}-\mathbf{e}_{i}^{M}, \mathbf{R}\right)+\sum_{d=1}^{D} \phi_{d}\left(R_{\max , d}-R_{d}\right) f\left(\mathbf{N}, \mathbf{R}+\mathbf{e}_{d}^{D}\right)+\sum_{d=1}^{D} \omega_{d} R_{d} \\
& \times G_{d}(f(\mathbf{N}, \mathbf{R}))+\left[\sum_{i=1}^{M} \mu_{i}\left(\left\lfloor\frac{R_{\max }}{r_{i}}\right\rfloor-N_{i}\right)+\sum_{d=1}^{D}\left(\phi_{d} R_{d}+\omega_{d}\left(R_{\max , d}-R_{d}\right)\right)\right] f(\mathbf{N}, \mathbf{R}) .
\end{aligned}
$$


Batur et al.: Dynamic Pricing of Wireless Internet

Article submitted to Manufacturing $\&$ Service Operations Management; manuscript no. (Please, provide the manuscript number!)B.1

Consider a function $f(\mathbf{N}, \mathbf{R})$ which is nonincreasing in $N_{j}, j=1, \ldots, M$. The image of this function under $T$ is given in Equation (6). Because $\lambda_{i}, i=1, \ldots, M$, are all positive, it is sufficient to show that $H_{j}(f(\mathbf{N}, \mathbf{R}))$ and $H_{k}(f(\mathbf{N}, \mathbf{R})), k=1, \ldots, M, j \neq k$, in Equation (4); $G_{d}(f(\mathbf{N}, \mathbf{R})), d=1, \ldots, D$, in Equation (5); and $H(\mathbf{N}, \mathbf{R})$ in Equation (B.2) are all nonincreasing in $N_{j}, j=1, \ldots, M$. Below, we only show how we study $H_{j}(f(\mathbf{N}, \mathbf{R}))$ and $G_{d}(f(\mathbf{N}, \mathbf{R}))$. We skip some of the details because the derivations are analogous.

We study $H_{j}(f(\mathbf{N}, \mathbf{R}))$ in the following two cases. i. $\left(\mathbf{N}+\mathbf{e}_{j}^{M}\right)^{\prime} \mathbf{r} \leq R<\left(\mathbf{N}+2 \mathbf{e}_{j}^{M}\right)^{\prime} \mathbf{r}$ and ii. $\left(\mathbf{N}+2 \mathbf{e}_{j}^{M}\right)^{\prime} \mathbf{r} \leq R$. In the second case, for example, we let $\ell_{2}=\arg \max _{\ell}\left[p_{j \ell}(f(\mathbf{N}+\right.$ $\left.\left.\left.2 \mathbf{e}_{j}^{M}, \mathbf{R}\right)+w_{j \ell}\right)+\left(1-p_{j \ell}\right) f\left(\mathbf{N}+\mathbf{e}_{j}^{M}, \mathbf{R}\right)\right]$. Then

$$
\begin{aligned}
& H_{j}\left(f\left(\mathbf{N}+\mathbf{e}_{j}^{M}, \mathbf{R}\right)\right)-H_{j}(f(\mathbf{N}, \mathbf{R})) \leq p_{j \ell_{2}}\left(f\left(\mathbf{N}+2 \mathbf{e}_{j}^{M}, \mathbf{R}\right)+w_{j \ell_{2}}\right)+\left(1-p_{j \ell_{2}}\right) f\left(\mathbf{N}+\mathbf{e}_{j}^{M}, \mathbf{R}\right) \\
& \quad-\left[p_{j \ell_{2}}\left(f\left(\mathbf{N}+\mathbf{e}_{j}^{M}, \mathbf{R}\right)+w_{j \ell_{2}}\right)+\left(1-p_{j \ell_{2}}\right) f(\mathbf{N}, \mathbf{R})\right] \\
& =\left(1-p_{j \ell_{2}}\right)\left(f\left(\mathbf{N}+\mathbf{e}_{j}^{M}, \mathbf{R}\right)-f(\mathbf{N}, \mathbf{R})\right)+p_{j \ell_{2}}\left(f\left(\mathbf{N}+2 \mathbf{e}_{j}^{M}, \mathbf{R}\right)-f\left(\mathbf{N}+\mathbf{e}_{j}^{M}, \mathbf{R}\right)\right) .
\end{aligned}
$$

The expression above is less than or equal to zero because $f$ is nonincreasing in $N_{j}$.

We study $G_{d}$ in the following three cases: i. $\left(\mathbf{N}+\mathbf{e}_{j}^{M}\right)^{\prime} \mathbf{r} \leq R-1$; ii. $R-1<\left(\mathbf{N}+\mathbf{e}_{j}^{M}\right)^{\prime} \mathbf{r}$ and $\mathbf{N}^{\prime} \mathbf{r} \leq R-1$; and iii. $R-1<\mathbf{N}^{\prime} \mathbf{r}$. In the third case, for example, we let

$$
\hat{\mathbf{N}}_{1}=\underset{\hat{\mathbf{N}}}{\arg \max }\left[f\left(\hat{\mathbf{N}}, \mathbf{R}-\mathbf{e}_{d}^{D}\right)-K \sum_{i=1, i \neq j}^{M}\left(N_{i}-\hat{N}_{i}\right)-K\left(N_{j}+1-\hat{N}_{j}\right)\right],
$$

where $\mathbf{N}^{\prime} \mathbf{r}>R-1, \hat{\mathbf{N}}^{\prime} \mathbf{r} \leq R-1, \hat{N}_{i} \leq N_{i}, i=1, \ldots, M, i \neq j$, and $\hat{N}_{j} \leq N_{j}+1$. We need to consider two sub-cases: i. $\hat{N}_{1 j}=N_{j}+1$ and ii. $\hat{N}_{1 j} \leq N_{j}$. When $\hat{N}_{1 j}=N_{j}+1$, we get

$$
\begin{aligned}
G_{d}( & \left.f\left(\mathbf{N}+\mathbf{e}_{j}^{M}, \mathbf{R}\right)\right)-G_{d}(f(\mathbf{N}, \mathbf{R})) \leq f\left(\hat{\mathbf{N}}_{1}, \mathbf{R}-\mathbf{e}_{d}^{D}\right)-K \sum_{i=1, i \neq j}^{M}\left(N_{i}-\hat{N}_{1 i}\right)-K\left(N_{j}+1-\hat{N}_{1 j}\right) \\
& -\left[f\left(\hat{\mathbf{N}}_{1}-\mathbf{e}_{j}^{M}, \mathbf{R}-\mathbf{e}_{d}^{D}\right)-K \sum_{i=1, i \neq j}^{M}\left(N_{i}-\hat{N}_{1 i}\right)-K\left(N_{j}-\left(\hat{N}_{1 j}-1\right)\right)\right] \\
= & f\left(\hat{\mathbf{N}}_{1}, \mathbf{R}-\mathbf{e}_{d}^{D}\right)-f\left(\hat{\mathbf{N}}_{1}-\mathbf{e}_{j}^{M}, \mathbf{R}-\mathbf{e}_{d}^{D}\right) .
\end{aligned}
$$

The expression above is less than or equal to zero because $f$ is nonincreasing in $N_{j}$.

When $\hat{N}_{1 j} \leq N_{j}$, we get

$$
G_{d}\left(f\left(\mathbf{N}+\mathbf{e}_{j}^{M}, \mathbf{R}\right)\right)-G_{d}(f(\mathbf{N}, \mathbf{R})) \leq f\left(\hat{\mathbf{N}}_{1}, \mathbf{R}-\mathbf{e}_{d}^{D}\right)-K \sum_{i=1, i \neq j}^{M}\left(N_{i}-\hat{N}_{1 i}\right)-K\left(N_{j}+1-\hat{N}_{1 j}\right)
$$




$$
\begin{aligned}
& -\left[f\left(\hat{\mathbf{N}}_{1}, \mathbf{R}-\mathbf{e}_{d}^{D}\right)-K \sum_{i=1}^{M}\left(N_{i}-\hat{N}_{1 i}\right)\right] \\
= & f\left(\hat{\mathbf{N}}_{1}, \mathbf{R}-\mathbf{e}_{d}^{D}\right)-K \sum_{i=1}^{M}\left(N_{i}-\hat{N}_{1 i}\right)-K-f\left(\hat{\mathbf{N}}_{1}, \mathbf{R}-\mathbf{e}_{d}^{D}\right)+K \sum_{i=1}^{M}\left(N_{i}-\hat{N}_{1 i}\right)=-K .
\end{aligned}
$$

Proof of Theorem 2,2. The proof is analogous to the previous proof.

Proof of Theorem 2.3. The statement can be proven by a sample path argument.

\section{Proof of Theorem 3}

Consider a function $f(\mathbf{N}, \mathbf{R})$ which satisfies Equation (9). The image of this function under $T$ is given in Equation (6). Because $\lambda_{i}$ are all positive, it is sufficient to show that $H_{i}(f(\mathbf{N}, \mathbf{R}))$ in Equation (4), $G_{d}(f(\mathbf{N}, \mathbf{R})), d=1, \ldots, D$, in Equation (5), and $H(\mathbf{N}, \mathbf{R})$ in Equation (B.2) also satisfy a relationship similar to Equation (9). Below, we only show how we study $H(\mathbf{N}, \mathbf{R})$. We skip some of the details because the derivations are analogous.

$$
\begin{aligned}
H & \left(\mathbf{N}+\mathbf{e}_{j}^{M}, \mathbf{R}\right)-H\left(\mathbf{N}+\mathbf{e}_{k}^{M}, \mathbf{R}\right) \\
= & \sum_{i=1, i \neq j, k}^{M} \mu_{i} N_{i}\left(f\left(\mathbf{N}+\mathbf{e}_{j}^{M}-\mathbf{e}_{i}^{M}, \mathbf{R}\right)-f\left(\mathbf{N}+\mathbf{e}_{k}^{M}-\mathbf{e}_{i}^{M}, \mathbf{R}\right)\right) \\
& +\sum_{d=1}^{D} \phi_{d}\left(R_{\max , d}-R_{d}\right)\left(f\left(\mathbf{N}+\mathbf{e}_{j}^{M}, \mathbf{R}+\mathbf{e}_{d}^{D}\right)-f\left(\mathbf{N}+\mathbf{e}_{k}^{M}, \mathbf{R}+\mathbf{e}_{d}^{D}\right)\right) \\
& +\sum_{d=1}^{D} \omega_{d} R_{d}\left(G_{d}\left(f\left(\mathbf{N}+\mathbf{e}_{j}^{M}, \mathbf{R}\right)\right)-G_{d}\left(f\left(\mathbf{N}+\mathbf{e}_{k}^{M}, \mathbf{R}\right)\right)\right) \\
& +\sum_{i=1, i \neq j, k}^{M} \mu_{i}\left(\left\lfloor\frac{R_{\max }}{r_{i}}\right\rfloor-N_{i}\right)\left(f\left(\mathbf{N}+\mathbf{e}_{j}^{M}, \mathbf{R}\right)-f\left(\mathbf{N}+\mathbf{e}_{k}^{M}, \mathbf{R}\right)\right) \\
& +\sum_{d=1}^{D}\left(\phi_{d} R_{d}+\omega_{d}\left(R_{\max , d}-R_{d}\right)\right)\left(f\left(\mathbf{N}+\mathbf{e}_{j}^{M}, \mathbf{R}\right)-f\left(\mathbf{N}+\mathbf{e}_{k}^{M}, \mathbf{R}\right)\right) \\
& +\mu_{k}\left(\left\lfloor\frac{R_{\max }}{r_{k}}\right\rfloor-\left(N_{k}+1\right)\right)\left(f\left(\mathbf{N}+\mathbf{e}_{j}^{M}, \mathbf{R}\right)-f\left(\mathbf{N}+\mathbf{e}_{k}^{M}, \mathbf{R}\right)\right) \\
& +\mu_{j}\left(\left\lfloor\frac{R_{\max }}{r_{j}}\right\rfloor-\left(N_{j}+1\right)\right)\left(f\left(\mathbf{N}+\mathbf{e}_{j}^{M}, \mathbf{R}\right)-f\left(\mathbf{N}+\mathbf{e}_{k}^{M}, \mathbf{R}\right)\right) \\
& +\mu_{k} N_{k}\left(f\left(\mathbf{N}+\mathbf{e}_{j}^{M}-\mathbf{e}_{k}^{M}, \mathbf{R}\right)-f(\mathbf{N}, \mathbf{R})\right)+\mu_{j} N_{j}\left(f(\mathbf{N}, \mathbf{R})-f\left(\mathbf{N}+\mathbf{e}_{k}^{M}-\mathbf{e}_{j}^{M}, \mathbf{R}\right)\right) \\
& +\mu_{j}\left(f\left(\mathbf{N}+\mathbf{e}_{j}^{M}, \mathbf{R}\right)-f\left(\mathbf{N}+\mathbf{e}_{k}^{M}, \mathbf{R}\right)\right)+\left(\mu_{k}-\mu_{j}\right)\left(f\left(\mathbf{N}+\mathbf{e}_{j}^{M}, \mathbf{R}\right)-f(\mathbf{N}, \mathbf{R})\right)
\end{aligned}
$$


Batur et al.: Dynamic Pricing of Wireless Internet
F.0Article submitted to Manufacturing \& Service Operations Management; manuscript no. (Please, provide the manuscript number!)

In the expression above, $\mu_{k} f\left(\mathbf{N}+\mathbf{e}_{j}^{M}, \mathbf{R}\right)$ and $\mu_{j} f\left(\mathbf{N}+\mathbf{e}_{k}^{M}, \mathbf{R}\right)$ are added and subtracted. The expression above is less than or equal to zero by Equation (9) when $\mu_{j} \leq \mu_{k}, N_{j}+1 \leq$ $\left\lfloor R_{\max } / r_{j}\right\rfloor$, and $N_{k}+1 \leq\left\lfloor R_{\max } / r_{k}\right\rfloor$.

\section{Proof of Theorem 4}

Assume that in state (N, R), a channel becomes unavailable. If the bandwidth-based dropoff policy recommends transitioning to state $\left(\mathbf{N}-\mathbf{c}, \mathbf{R}-\mathbf{e}_{d}^{D}\right)$ where $\mathbf{c} \in \mathbb{Z}_{+}^{M}$, the following must hold: i. $\mathbf{N}^{\prime} \mathbf{r}>R-1$; ii. $(\mathbf{N}-\mathbf{c})^{\prime} \mathbf{r} \leq R-1$; and iii. $\left(\mathbf{N}-\mathbf{c}+\mathbf{e}_{i}^{M}\right)^{\prime} \mathbf{r}>R-1$ for all $i=1, \ldots, M$. These imply that at least $c$ customers must be dropped. If the optimal drop-off policy recommends transitioning to state $\left(\mathbf{N}-\hat{\mathbf{c}}, \mathbf{R}-\mathbf{e}_{d}^{D}\right)$, then $\hat{c}<c$ cannot hold.

Next, we will prove by contradiction that $\hat{c}>c$ cannot hold. Assume that $\hat{c}>c$. According to the optimal drop-off function $G_{d}$ in Equation $(5), v\left(\mathbf{N}-\hat{\mathbf{c}}, \mathbf{R}-\mathbf{e}_{d}^{D}\right)-K \hat{c} \geq v(\mathbf{N}-\mathbf{c}, \mathbf{R}-$ $\left.\mathbf{e}_{d}^{D}\right)-K c$ must hold. This is equivalent to $v\left(\mathbf{N}-\mathbf{c}, \mathbf{R}-\mathbf{e}_{d}^{D}\right)-v\left(\mathbf{N}-\hat{\mathbf{c}}, \mathbf{R}-\mathbf{e}_{d}^{D}\right)+K(\hat{c}-c) \leq 0$. This result contradicts Lemma B1 when $\hat{c}>c$. Hence, $\hat{c}>c$ cannot hold. As a result of these, we conclude that $\hat{c}=c$ must hold.

\section{E. Proof of Theorem 6}

Proof of Theorem 6.1. Suppose that the arrival rate for class $m$ is changed from $\lambda_{m}$ to $\hat{\lambda}_{m}, \hat{\lambda}_{m}>\lambda_{m}$. Define $T\left(\lambda_{m}\right)$ and $T\left(\hat{\lambda}_{m}\right)$ as the dynamic programming operators. Let $v\left(N, \mathbf{R}, \lambda_{m}\right)$ and $v\left(N, \mathbf{R}, \hat{\lambda}_{m}\right)$ be the corresponding solutions to the MDP optimality equation, so that $v\left(N, \mathbf{R}, \lambda_{m}\right)=T\left(\lambda_{m}\right) v\left(N, \mathbf{R}, \lambda_{m}\right)$ and $v\left(N, \mathbf{R}, \hat{\lambda}_{m}\right)=T\left(\hat{\lambda}_{m}\right) v\left(N, \mathbf{R}, \hat{\lambda}_{m}\right)$. We will show that

$$
v\left(N+1, \mathbf{R}, \hat{\lambda}_{m}\right)-v\left(N, \mathbf{R}, \hat{\lambda}_{m}\right) \leq v\left(N+1, \mathbf{R}, \lambda_{m}\right)-v\left(N, \mathbf{R}, \lambda_{m}\right)
$$

This would imply that the optimal discounted price for a class $i$ customer, $w_{i}^{*}(N, \mathbf{R})$, is a nondecreasing function of the arrival rate $\lambda_{j}, j=1, \ldots, M$, due to Theorem 1 .

We define the sequence of approximations for the optimal discounted profit function $v_{q}\left(N, \mathbf{R}, \hat{\lambda}_{m}\right), q \geq 0$, such that $v_{q}\left(N, \mathbf{R}, \hat{\lambda}_{m}\right)=T\left(\hat{\lambda}_{m}\right) v_{q-1}\left(N, \mathbf{R}, \hat{\lambda}_{m}\right), q \geq 1$, and $v_{0}\left(N, \mathbf{R}, \hat{\lambda}_{m}\right)=v\left(N, \mathbf{R}, \lambda_{m}\right)$. Given the contracting nature of $T\left(\hat{\lambda}_{m}\right), v\left(N, \mathbf{R}, \hat{\lambda}_{m}\right)=$ $\lim _{q \rightarrow \infty} v_{q}\left(N, \mathbf{R}, \hat{\lambda}_{m}\right)$. Thus, Equation E.1 will be proven if we show that $v_{q+1}(N+$ $\left.1, \mathbf{R}, \hat{\lambda}_{m}\right)-v_{q+1}\left(N, \mathbf{R}, \hat{\lambda}_{m}\right) \leq v_{q}\left(N+1, \mathbf{R}, \hat{\lambda}_{m}\right)-v_{q}\left(N, \mathbf{R}, \hat{\lambda}_{m}\right)$. This can be proven by induction for which the details we skip because of space limitations.

The proofs of Theorems 6,2 and 6.3 are analogous to the proof of Theorem 6.1. 


\section{F. Proof of Theorem 7}

Suppose by contradiction that $w_{j}^{*}(N, \mathbf{R})<w_{k}^{*}(N, \mathbf{R})$ for some $(N, \mathbf{R})$. Let $\ell_{1}=$ $\arg \max _{\ell}\left[p_{j \ell}\left(f(N+1, \mathbf{R})+w_{\ell}\right)+\left(1-p_{j \ell}\right) f(N, \mathbf{R})\right]$ and $\ell_{2}=\arg \max _{\ell}\left[p_{k \ell}(f(N+1, \mathbf{R})+\right.$ $\left.\left.w_{\ell}\right)+\left(1-p_{k \ell}\right) f(N, \mathbf{R})\right]$. Then let $w_{j}^{*}(N, \mathbf{R})=w_{\ell_{1}}$ and $w_{k}^{*}(N, \mathbf{R})=w_{\ell_{2}}$. When $w_{\ell_{1}}<w_{\ell_{2}}$, $\ell_{1}<\ell_{2}$ by definition; and $p_{j \ell_{1}}>p_{j \ell_{2}}$ and $p_{k \ell_{1}}>p_{k \ell_{2}}$ by Assumption 2, From the definition of $\ell_{1}$, we get $p_{j \ell_{1}}\left(f(N+1, \mathbf{R})-f(N, \mathbf{R})+w_{\ell_{1}}\right) \geq p_{j \ell_{2}}\left(f(N+1, \mathbf{R})-f(N, \mathbf{R})+w_{\ell_{2}}\right)$; and then because $p_{j \ell_{1}}>p_{j \ell_{2}}$, we have

$$
f(N, \mathbf{R})-f(N+1, \mathbf{R}) \leq\left(p_{j \ell_{1}} w_{\ell_{1}}-p_{j \ell_{2}} w_{\ell_{2}}\right) /\left(p_{j \ell_{1}}-p_{j \ell_{2}}\right) .
$$

From the definition of $\ell_{2}$, we get $p_{k \ell_{2}}\left(f(N+1, \mathbf{R})-f(N, \mathbf{R})+w_{\ell_{2}}\right) \geq p_{k \ell_{1}}(f(N+1, \mathbf{R})-$ $\left.f(N, \mathbf{R})+w_{\ell_{1}}\right)$; and then because $p_{k \ell_{1}}>p_{k \ell_{2}}$, we have

$$
f(N, \mathbf{R})-f(N+1, \mathbf{R}) \geq\left(p_{k \ell_{1}} w_{\ell_{1}}-p_{k \ell_{2}} w_{\ell_{2}}\right) /\left(p_{k \ell_{1}}-p_{k \ell_{2}}\right)
$$

From Equations (F.1)- F.2), we get $\left(p_{k \ell_{1}} w_{\ell_{1}}-p_{k \ell_{2}} w_{\ell_{2}}\right) /\left(p_{k \ell_{1}}-p_{k \ell_{2}}\right) \leq\left(p_{j \ell_{1}} w_{\ell_{1}}-\right.$ $\left.p_{j \ell_{2}} w_{\ell_{2}}\right) /\left(p_{j \ell_{1}}-p_{j \ell_{2}}\right)$, which implies $p_{j \ell_{2}} / p_{k \ell_{2}} \leq p_{j \ell_{1}} / p_{k \ell_{1}}$. This contradicts the given condition in the paper which says $p_{j \ell_{2}} / p_{k \ell_{2}}>p_{j \ell_{1}} / p_{k \ell_{1}}$ when $\ell_{1}<\ell_{2}$. Hence, $w_{j}^{*}(N, \mathbf{R}) \geq w_{k}^{*}(N, \mathbf{R})$ for all $(N, \mathbf{R})$ must hold.

\section{G. Sensitivity Analysis of the Optimal Policy}

Here, we present sensitivity analysis results for the optimal average values, $\bar{v}$, obtained from the VIA in Experiment 1. In Figure G.1, we show how the normalized optimal values change as each sensitivity parameter, $\Delta_{j}$, changes. We only show 18 representative lines out of the 729 configurations. The remaining lines show similar behavior. Here, normalization of the optimal value of a configuration is performed with respect to the optimal value of the configuration in which the relevant $\Delta_{j}$ (i.e., the $\Delta_{j}$ shown in the $x$-axis label) is set to its smallest value. For example, in Figure $1(\mathrm{a})$, the normalized $\bar{v}$ is $\left(\bar{v}_{\mathrm{c}}-\bar{v}_{\mathrm{b}}\right) / \bar{v}_{\mathrm{b}}$, where $\Delta_{1} \in\{0.25,0.5,1\}$ for configuration $c$ and $\Delta_{1}=0.25$ for configuration $b$. The purpose of the normalization is to have all lines start from the same origin in the same figure. This way, we can more clearly see how each line behaves as $\Delta_{j}$ changes. The results are consistent with the structural results proven in Section 4. 


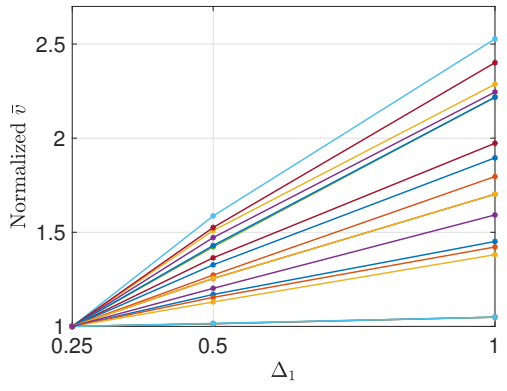

(a) $\frac{r_{2}}{r_{1}}$

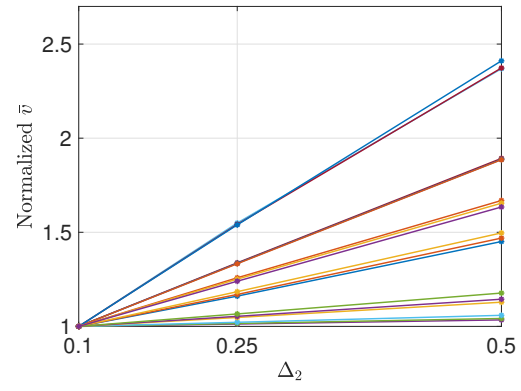

(b) $\frac{\lambda_{2}}{\lambda_{1}}$

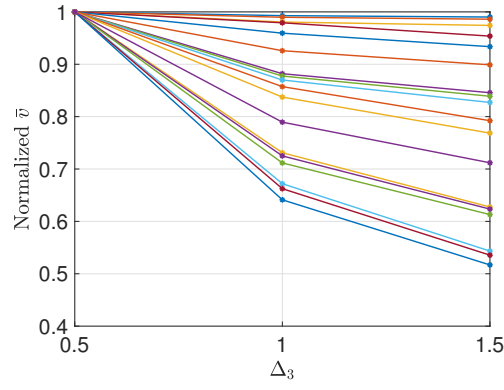

(c) $\frac{\mu_{2}}{\mu_{1}}$

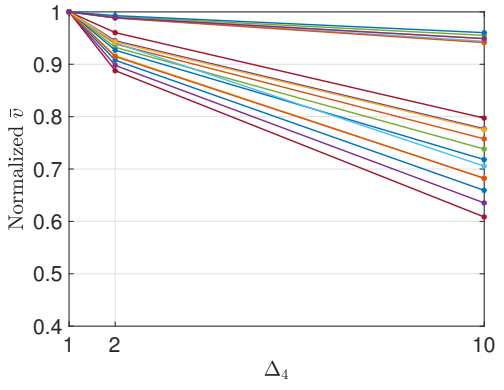

(d) $K$

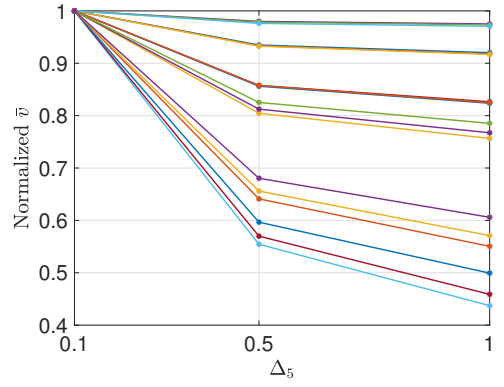

(e) $\frac{\beta_{2}}{\beta_{1}}$

Figure G.1 Sensitivity analysis of the normalized optimal average values from the VIA in Experiment 1

\section{H. Sensitivity Analysis of the Proposed Heuristic Policies}

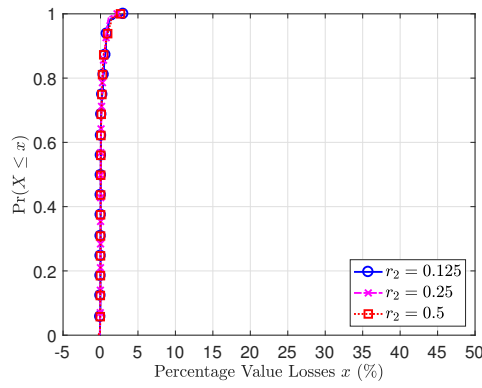

(a) $R$-heuristic, $r_{2}$

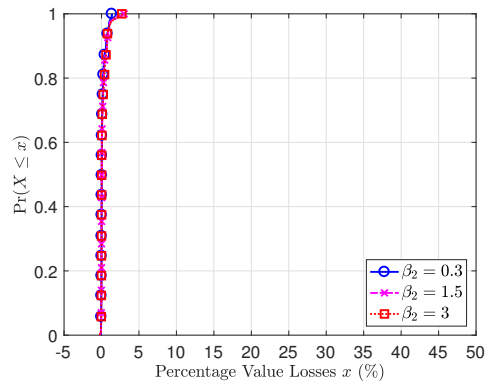

(d) $R$-heuristic, $\beta_{2}$

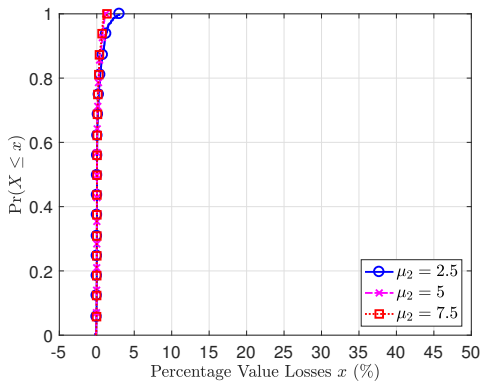

(b) $R$-heuristic, $\mu_{2}$

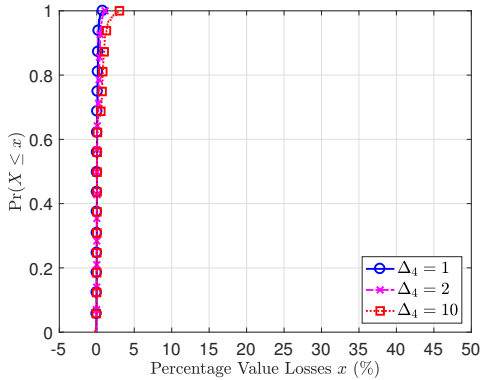

(e) $R$-heuristic, $K\left(\Delta_{4}\right)$

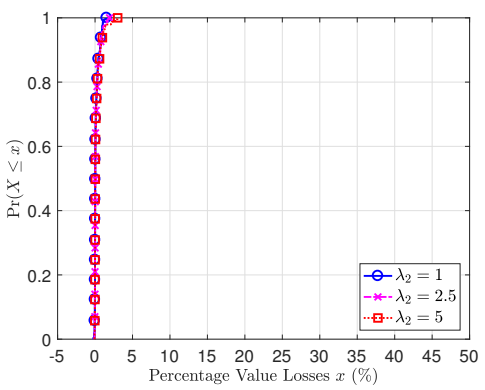

(c) $R$-heuristic, $\lambda_{2}$

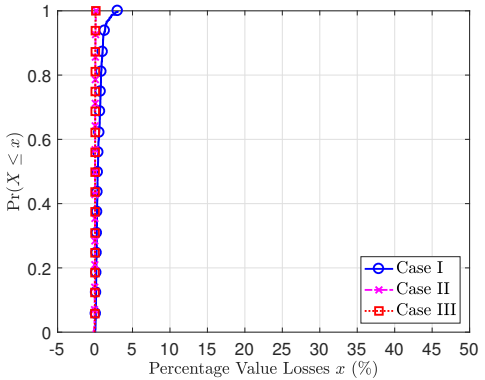

(f) $R$-heuristic, channel-class case

Figure H.1 Empirical CDFs of the percentage value loss of the $R$-heuristic categorized by $r_{2}, \mu_{2}, \lambda_{2}, \beta_{2}, K$, and channel-class case (across 40 replications in Experiment 1). 
Batur et al.: Dynamic Pricing of Wireless Internet

Article submitted to Manufacturing \& Service Operations Management; manuscript no. (Please, provide the manuscript number!)H.1

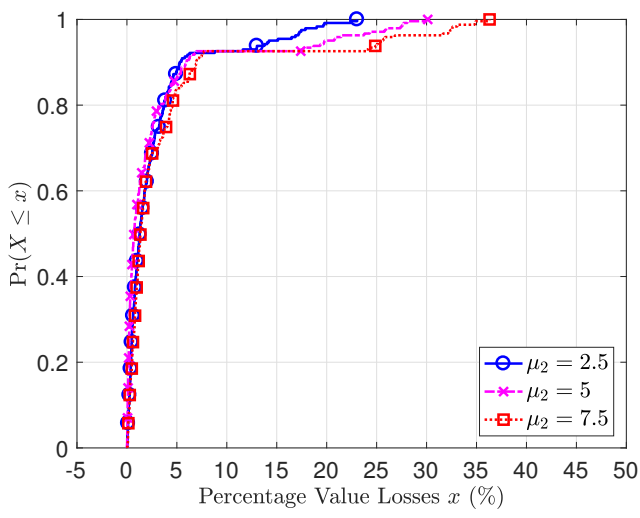

(a) $N$-heuristic, $\mu_{2}$

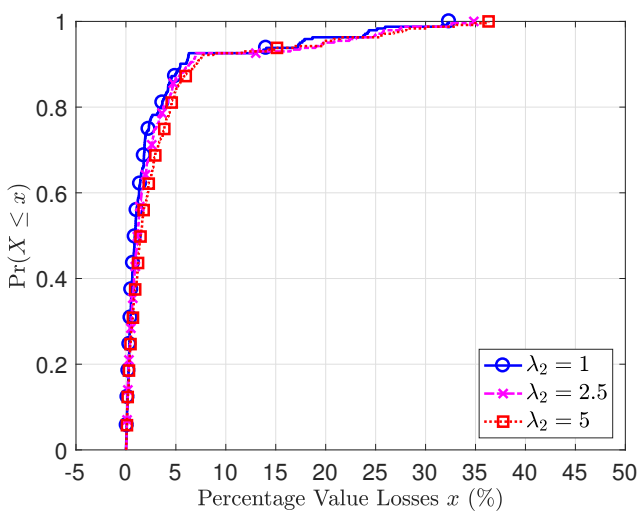

(c) $N$-heuristic, $\lambda_{2}$

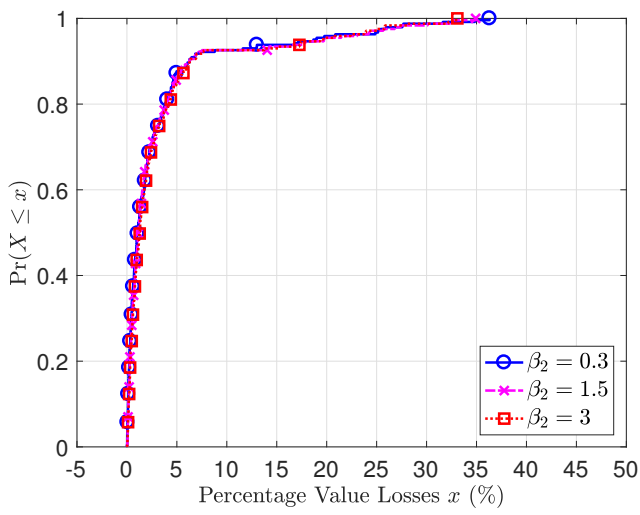

(e) $N$-heuristic, $\beta_{2}$

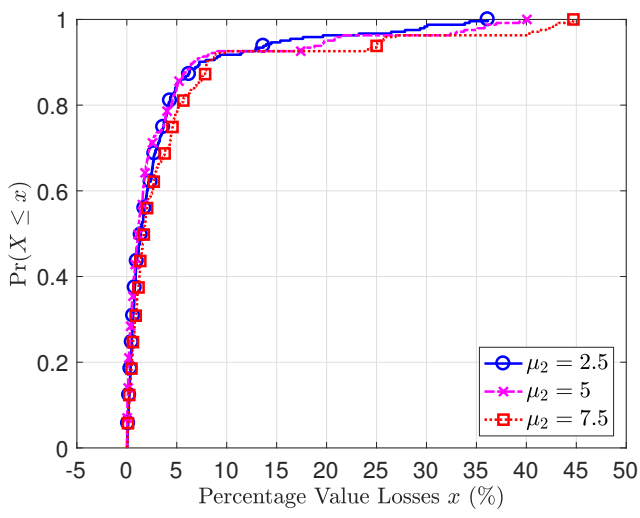

(b) $(N, R)$-heuristic, $\mu_{2}$

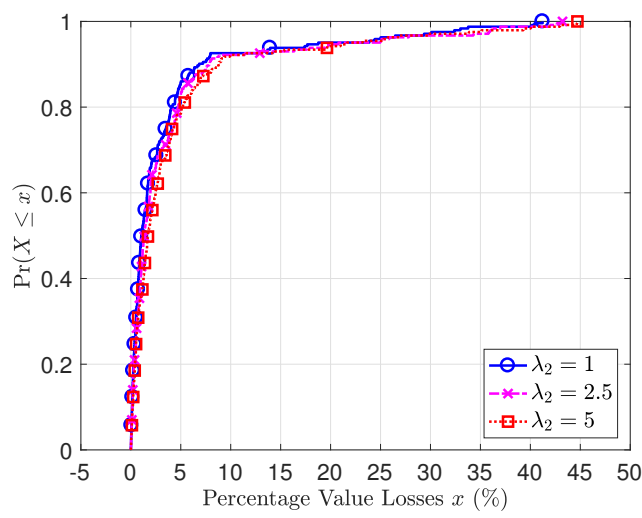

(d) $(N, R)$-heuristic, $\lambda_{2}$

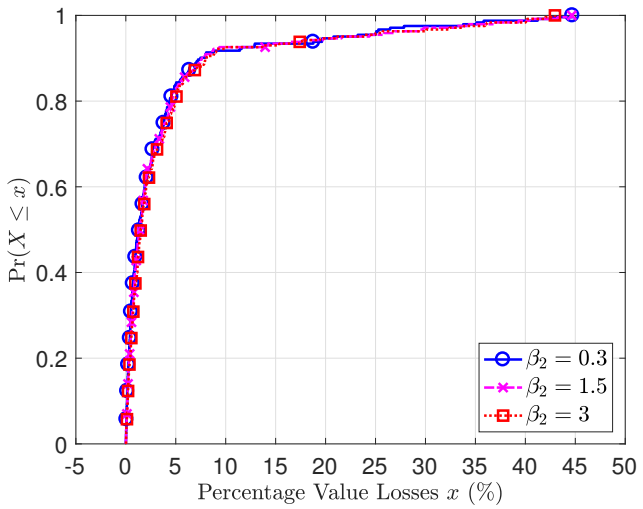

(f) $(N, R)$-heuristic, $\beta_{2}$

Figure H.2 Empirical CDFs of the percentage value loss of the $N$ - and $(N, R)$-heuristics categorized by $\mu_{2}, \lambda_{2}$, and $\beta_{2}$ (across 40 replications in Experiment 1). 


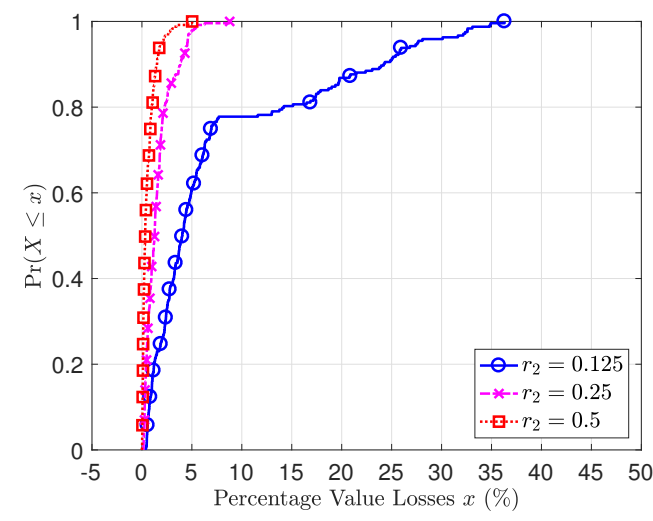

(a) $N$-heuristic, $r_{2}$

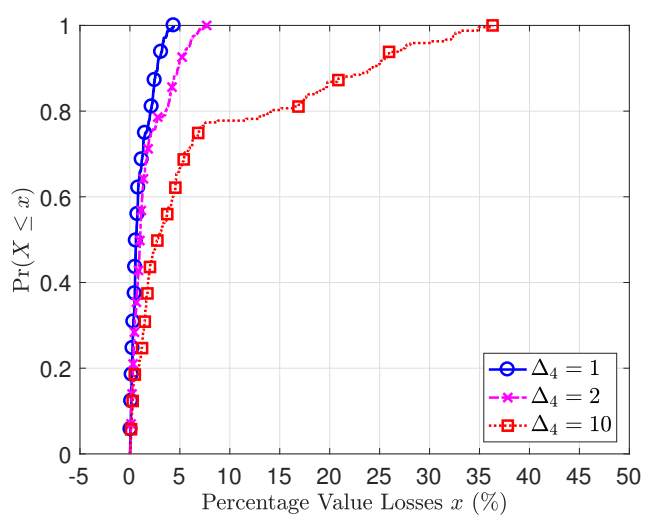

(c) $N$-heuristic, $K$

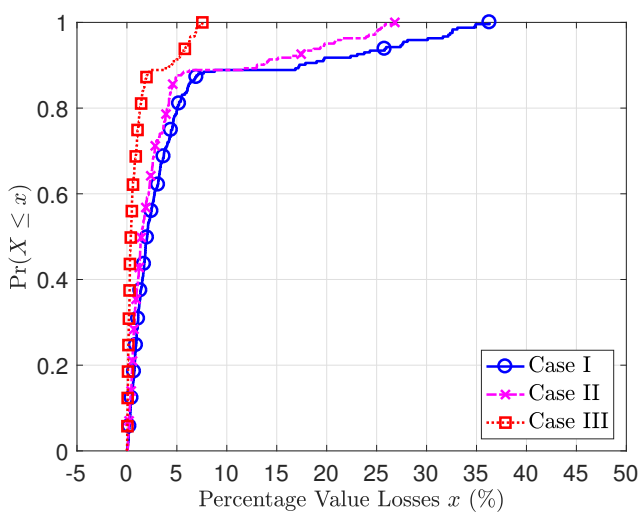

(e) $N$-heuristic, channel-class case

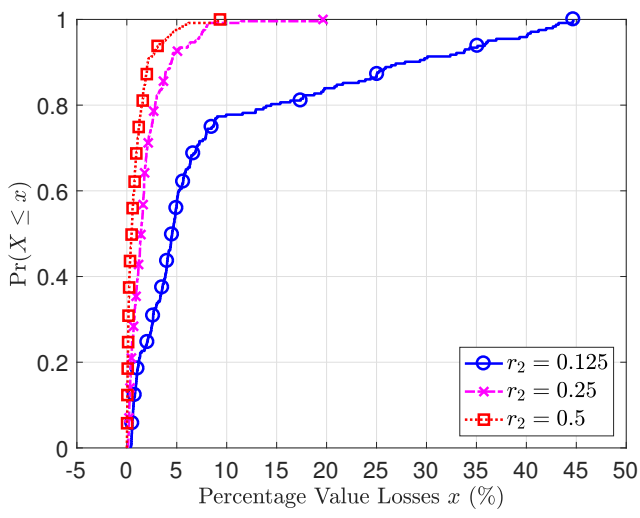

(b) $(N, R)$-heuristic, $r_{2}$

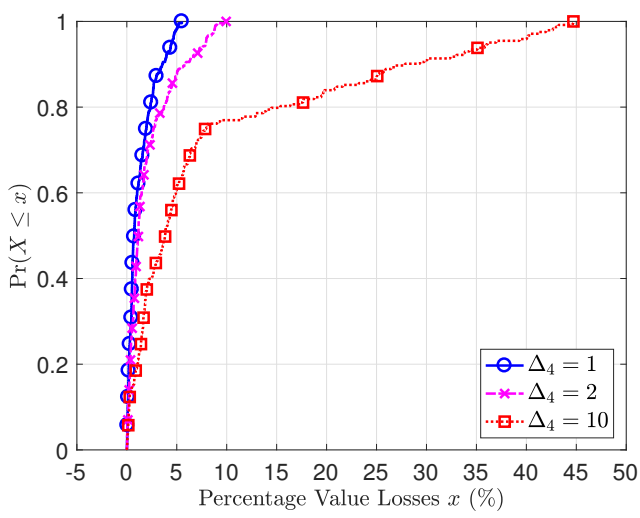

(d) $(N, R)$-heuristic, $K$

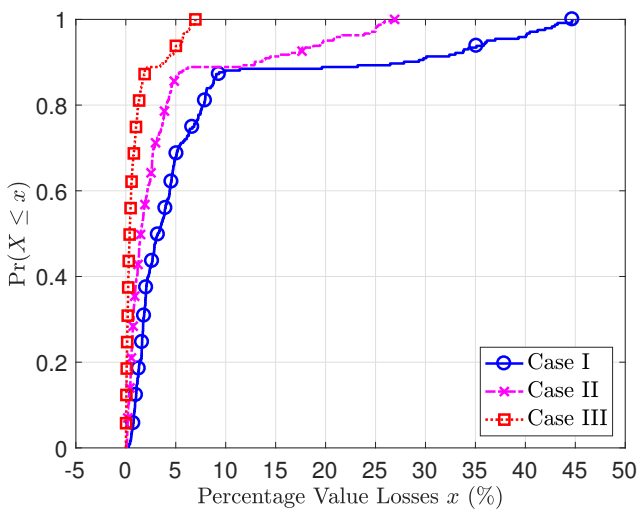

(f) $(N, R)$-heuristic, channel-class case

Figure H.3 Empirical CDFs of the percentage value loss of the $N$ - and $(N, R)$-heuristics categorized by $r_{2}, K$, and channel-class case (across 40 replications in Experiment 1). 